\title{
Campaign to Minimize the Transverse Impedance of the DARHT-2 Induction Linac Cells
}

\author{
Authors* \\ Richard Briggs (SAIC) \\ William Fawley (LBNL) \\ Richard Briggs (SAIC) \\ Daniel Birx (deceased) \\ William M. Fawley (LBNL) \\ Timothy Houck (LLNL) \\ Scott Nelson (LLNL) \\ Louis Reginato (LBNL) \\ Michael Vella (LBNL) \\ Glen Westenskow (LLNL)
}

Report content based upon contributions from*:

* The authors are responsible for the contents and conclusions in this report. 


\title{
Campaign to Minimize the Transverse Impedance of the DARHT-2 Induction Linac Cells
}

\begin{abstract}
$\underline{\text { ABSTRACT }}$
This paper discusses the multi-year "campaign" to measure and reduce the transverse impedances of the original DARHT-2 accelerator cells in order to limit the beam-breakup instability (BBU) growth through the machine. A series of three prototype cells were designed, modeled, and measured. One of the most important methods for limiting the impedance was the introduction of ferrite absorbers in the oil region immediately upstream of the insulator. When properly "tuned" in terms of thickness, the effective $Q$ 's could be significantly reduced for both the 10" "standard" cells and the 14" "injector" cells.

We also present a new experimental method ("twin lead-loop") to measure the transverse impedance. This method has a number of important advantages over the well-known TSD approach, especially in the low $Q$ regime. For the final prototype standard cell design, we found that $Z_{\perp}$ could be limited to $<300 \mathrm{ohms} / \mathrm{m}$ with $Q$ 's ranging from 3 to 6 . There was reasonable agreement between measurements and simulation results from the AMOS code (peak amplitudes within 25-30\%) with the exception that the resonant mode around $200 \mathrm{MHz}$ had a double-peaked structure that could not be reproduced by simulation.
\end{abstract}

\section{Introduction}

During the design of the original DARHT-2 accelerator cells, a very high priority was placed on minimizing the resistive part of the transverse impedance that controls the beam breakup instability (BBU) growth. Minimal transverse displacements (and excellent emittance) are required to produce the small spot sizes on a conversion target needed for high quality radiography. The 2-microsecond pulse length together with beam currents of 2-4 kA make high $Q$ transverse modes especially dangerous in this machine as compared to short pulse induction linacs like the DARHT first axis accelerator. The stringent high vacuum requirements that follow both from this long pulse length and the utilization of thermionic cathodes, together with the necessary robustness to electrical breakdown damage, made it desirable (if not mandatory!) to use ceramic insulators with their high dielectric constant. Impedance mismatches in the gap region leading to high $Q^{\prime}$ s of the resonant modes were therefore a major concern initially. Another rationale for high vacuum arises from the requirement to limit ion hose instability growth, also made dangerous by the long pulse length.

A campaign combining theoretical modeling and transverse impedance measurements of test cells and early prototypes was mounted early in the project to optimize the accelerator cell design for minimal BBU growth. A synopsis of this campaign and its path to the final design choices is presented in Section 2. 
An important byproduct of this campaign was the development of a new technique for measuring the broadband transverse impedance of heavily damped induction accelerator cells. Previously developed techniques, such as TSD and the measurement of frequency shifts of resonant modes to determine $Z_{\perp} / Q$, were used in some of the initial studies. These techniques were found to be very difficult and/or inadequate for a final determination of the complex impedance of these massive, heavily damped cells over the full frequency band of interest in the several meter wavelength region. This was especially true for impedance measurements of the 14"-diameter bore injector cells. The new technique is described in Section 3. It uses excitation of the cell by a twin lead running down the axis, as in the TSD approach, but the impedance is deduced from ratios of magnetic RF loop signals inside the accelerator gap and near the beam tube wall some distance away from the gap. In Section 4, transverse impedance measurements of both the "standard" (10-inch beam pipe diameter) and "injector" (14-inch diameter) production cells with this new technique are presented.

\section{Synopsis of the Campaign to Minimize the Cell Impedance}

A description of the original DARHT-2 accelerator design is presented in Ref. [1]. The first block of 8 accelerator cells (the so-called "injector" cells) in the linac have a 14-inch diameter bore beam tube. The remaining 72 accelerator cells (the "standard cells") have a 10-inch bore beam tube. The larger bore was chosen for the initial 8 cells for several reasons:

- It helps minimize beam spill of the off-energy electrons in the beam head, which could lead to voltage-holding problems and/or ion production that will disrupt beam transport.

- It reduces the transverse impedance (through the $1 / b^{2}$ algebraic factor discussed below) in a region where the focusing solenoidal field is quite low (which was necessary to smoothly match the beam from the injector region to the rest of the accelerator).

- It shifts the values of resonant frequencies of the BBU transverse modes (the peaks in the resistive impedance) away from those corresponding to the standard cells.

A cutaway view of the final design of the DARHT-2 standard cell containing its four Metglas cores is shown in Fig. 1. Four current feeds, separated azimuthally by 90 degrees, deliver the power pulse to the radial line connected to the accelerator gap. A ceramic insulator separates the vacuum in the beamline and accelerator

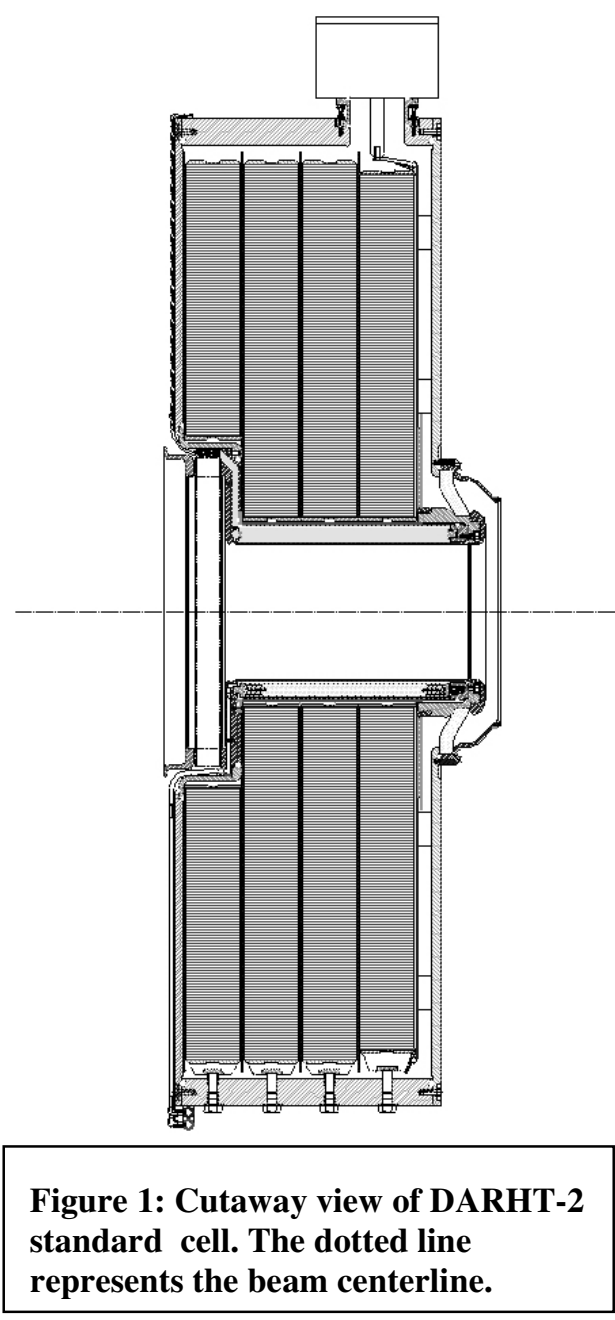


gap region from the oil insulation region containing the Metglas. Focusing solenoids are located just inside the beam tube as shown.

The goals for maximum allowable transverse impedance follow directly from the DARHT-2 project requirement that at the accelerator output location the transverse oscillation amplitude be less than $10 \%$ of the beam radius (over the frequency band 50 $\mathrm{MHz}$ to $2 \mathrm{GHz}$ ). As a reference point, the analytic estimate of BBU gain (i.e. the number of e-foldings) through $N$ accelerator cells is

$$
G=\frac{N I_{b}}{B c} Z_{\perp r}
$$

where $Z_{\perp r}$ is the resistive component of the transverse impedance of one cell (in ohms/meter), $I_{b}$ is the beam current, $c$ is the speed of light, and $B$ is the solenoid focusing field. (An average of $1 / B$ should be used to compute the gain over regions where this ratio varies substantially). For the original DARHT-2 configuration with 72 standard cells and a typical focus field of order $1 \mathrm{kG}$, this analytic estimate predicts three e-folds of growth (20X amplification) with a resistive impedance of $300 \mathrm{ohms} / \mathrm{m}$ and a beam current of $4 \mathrm{kA}$. An initial "benchmark" goal for the impedance of the standard cells was therefore set at $300 \mathrm{ohms} / \mathrm{m}$. It was felt that this value would provide an adequate safety margin for the baseline $2 \mathrm{kA}$ beam current, with a reasonable expectation that the accelerator could be upgraded to $4 \mathrm{kA}$ without any changes to the cells.

Since the solenoidal focusing field through the 8 injector cells is of order 200 gauss for typical beam dynamics tunes, the value of $N / B$ here is $\sim 50 \%$ of the $N / B$ in the rest of the accelerator. So minimization of the injector cell impedance is still important even though there are far fewer of these cells. A goal for the impedance of these cells of around $200 \mathrm{ohms} / \mathrm{m}$ was felt to be reasonable considering their larger diameter.

To avoid keeping the reader in suspense, we note that the impedance minimization campaign was highly successful: as detailed in Section 3, the final measured impedance of the production standard cell was $280 \mathrm{ohm} / \mathrm{m}$ and the production injector cell was 160 ohms/m.

\subsection{Main Design Variables Used for Minimizing the Transverse Impedance}

Previous studies of the transverse impedance of heavily damped induction cells have introduced a dimensionless "form factor" of order unity $\eta_{f}$ through the definition

$$
Z_{\perp r}=\sqrt{\frac{\mu_{0}}{\varepsilon_{0}}} \frac{w}{\pi b^{2}} \eta_{f}
$$

The above expression illustrates that the fundamental geometrical scaling factor in the transverse impedance is $w / b^{2}$ where $w$ is the (shortest distance) across the accelerator gap and $b$ is the beam pipe radius. In Section 3, we will derive this expression for the transverse impedance and give a simple physical interpretation of the form factor $\eta_{f}$, including showing how it can be measured directly. 
The peak electric field stress on the negative electrode of the accelerator gap constrains the choice of $w$. For DARHT-2, a value of $2.54 \mathrm{~cm}$ (i.e. 1.0 inch) was chosen, corresponding to a stress of $85 \mathrm{kV} / \mathrm{cm}$ at the nominal operating voltage of $193 \mathrm{kV}$ in the standard cells. The accelerating voltage gradient in an induction linac and its cost are adversely affected by increasing the inner bore. The compromise chosen for the standard cell (the majority of the DARHT-2 cells) was $b=5$ inches. As noted above, the first 8 cells have a larger bore in part because their relatively low focusing field makes BBU growth rates much larger. The geometrical choices for $w$ and $b$ were fixed at the outset of the DARHT-2 design and were considered inalterable during the campaign to minimize the impedance.

In contrast to RF linacs, an induction cell does not need to support a high $Q$ acceleration mode. Placing ferrite damping material in strategic locations in the cell to lower the $Q$ 's of all transverse modes to very low values has therefore become standard practice in the design of high current electron induction accelerators. A major focus of our experimental and theoretical campaign, particularly in the early phases, was to determine where the placement of ferrite tiles would be most effective in damping the resonant BBU modes. We also made judicious choices of ferrite tile thickness to minimize wave reflection over the most important frequency band. This sensitivity to thickness is a well-known feature of UHF ferrite absorbers ("thinner is sometimes better!"). In general, the utilization and optimization of ferrite dampers is the most powerful technique that one can use to minimize the transverse interaction impedance in induction linacs.

Another geometrical feature that can be exploited to minimize the impedance is adjustment of the shape of the conductors in the vacuum region immediately adjacent to the insulator. The importance of this was shown many years ago in ATA accelerator cell impedance studies [2]. In the ATA cell, the insulator was located between the outer cylindrical walls just beyond the outer radius of the radial line. Purposefully angling the radial conducting wall facing the insulator at the Brewster angle (in that case) significantly reduced wave reflections from the insulator. This "corner reflector" then allowed the outgoing radial wave to be absorbed better by ferrite dampers placed behind the insulator on the drive blades and the back wall, thereby dramatically lowering the $Q$ 's of the transverse BBU modes. This same general feature was found to be an important variable to optimize in the DARHT-2 accelerator cell as well.

\subsection{Initial Studies with Test Cells}

For DARHT-2, we expected that the transverse mode structure and cell impedance would be dominated by the region comprised of the beam tube, accelerator gap, insulator, and radial line connecting the gap with the pulse power feeds

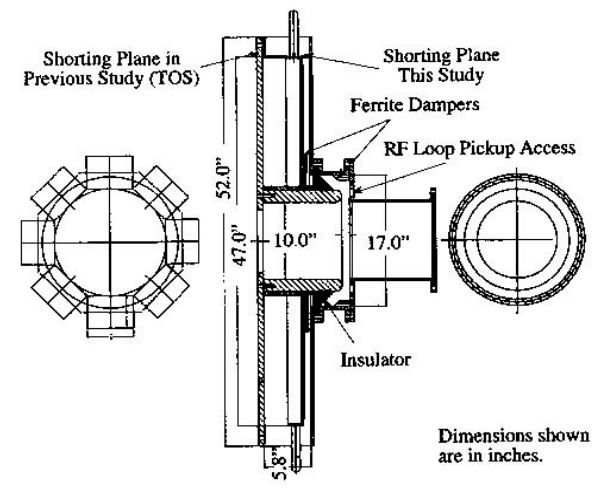
lines. To evaluate various placements of ferrite damping material and the effect of insulator dielectric constant before cells

Figure 2: Illustration of the long pulse Test Cell used in the DARHT-2 TOS study which shows the shorting rings and damping ferrites. 


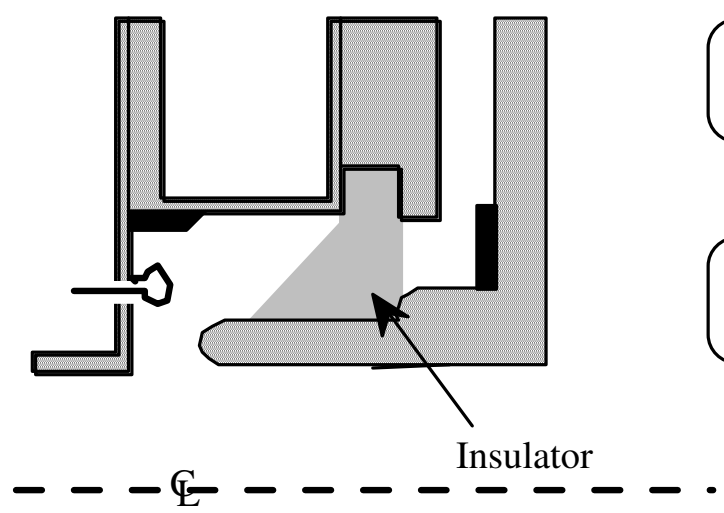

Ferrite Dampers

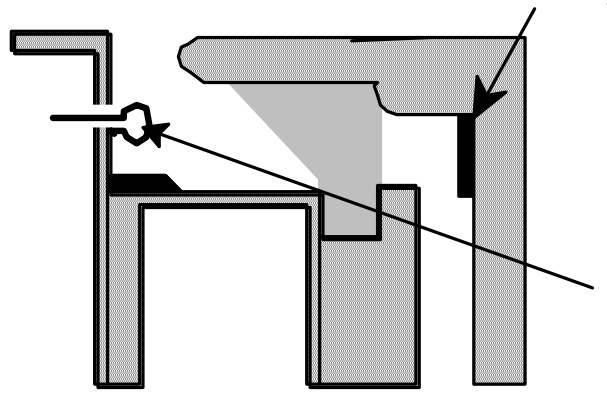

Gap - Side View

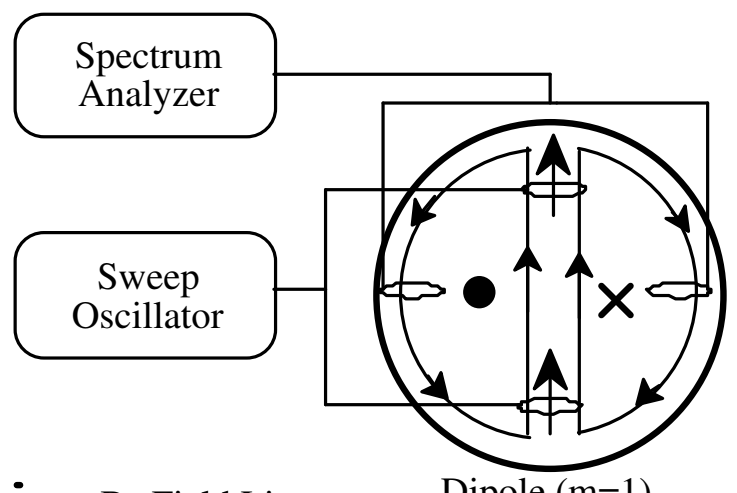

Dipole $(\mathrm{m}=1)$

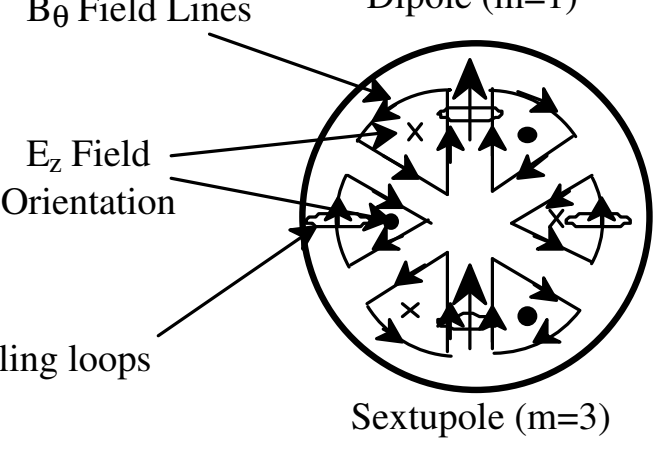

Gap - End View

Figure 3: Schematic of $\mathbf{B}_{\theta}$ coupling loops, orientation of electromagnetic fields relative to loops, and cavity excitation technique.

with the Metglas were available, a test cell without Metglas was constructed (see Fig. 2). In the first preliminary experiments, during the "Technology Options Study" (TOS) in 1996-97, a Rexolite insulator (dielectric constant around 2.7) was used and the outer "coax" was terminated with a shorting plane about 6 inches from the outer radial line plate (see Fig. 2). A follow-on study used the same test cell with an insulator made from an epoxy mix to simulate a ceramic insulator with dielectric constant around 6.9. The main objective of this second study was to determine whether a high dielectric constant insulator would compromise the suppression of transverse BBU modes with ferrite dampers, and to get preliminary indications on where these dampers should be placed in the cell. In the second study, a shorting ring was placed at the outer radius of the radial line to make better electrical contact. In both cases, beam tube sections about one tube diameter long were placed on both sides of the acceleration gap to ensure that the fields of the modes below cutoff have decayed to small levels by the end of the pipe.

The approach used in these impedance measurements was similar to that used 20 years ago by Dan Birx in his studies of the ETA and ATA cavities (Ref. [3]). RF-coupling loops were inserted in the cell in the vicinity of the accelerating gap as shown in Fig. 3. A sweep oscillator drove a pair of balanced loops spaced 180 degrees azimuthally, and oriented in the radial direction with an "up-up" polarity as shown. A spectrum analyzer picked up the signal from a similar set of balanced loops, separated by 90 degrees in azimuth from the drive loops, oriented in the theta direction with an "up-up" polarity. This excitation and pickup technique discriminated against modes other than dipole (and sextupole plus higher order odd mode numbers). 
Figure 4 displays measurements over a frequency range from 150 to $650 \mathrm{MHz}$ of the RF loop pickup in an undamped cell with the high dielectric constant insulator. The resonant modes in the cavity are clearly evident with dipole modes identified as the TM110, TM120, and TM130 indicated on the plot. A response indicated as "A" at about $500 \mathrm{MHz}$ is interpreted as a sextupole mode. Sextupole modes are also excited and detected with this setup, as explained in the illustration in Fig. 3. An experimental check for this interpretation was provided by looking for a frequency shift in this resonance when a 4-inch diameter metal rod was inserted on axis. As there was no measurable frequency shift, this indicates that there was minimal magnetic field energy on axis, consistent with a sextupole mode. MAFIA code simulations also predicted

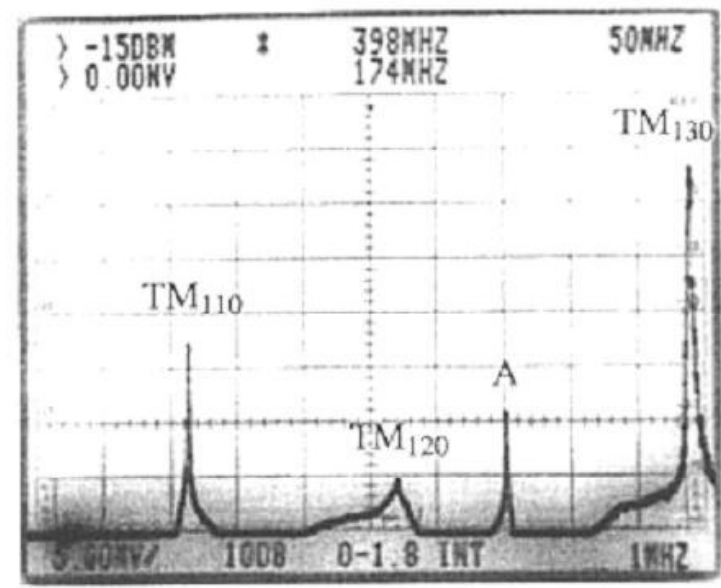

Figure 4: Loop pickup data in the undamped cell for the orientation of the drive loop and pickup orientation illustrated in Fig. 3. The oscillogram has $50 \mathrm{MHz} / \mathrm{div}$. and a center frequency of $398 \mathrm{MHz}$. that the lowest sextupole mode frequency was $\sim 505 \mathrm{MHz}$.

The $Q$ of the dipole modes in the test cell without any ferrite dampers was high enough to use frequency shift techniques to infer $Z / Q$. By inserting cylindrical metal rods of various diameters and lengths in the beam pipe, the resultant shift in resonant frequency could be measured and related to $Z / Q$ using the Slater perturbation formula (Ref. [4]). Table 1 summarizes the results along with the measured resonant frequencies. The eigenmode solver module of MAFIA was used to calculate the resonant frequencies and $Z / Q$ for this test cell; these results are also given in Table 1. The agreement is reasonable, as might be expected for this "simple" electromagnetic configuration. The TM 130 mode was too close to the pipe cutoff frequency of $690 \mathrm{MHz}$ to make meaningful interpretations of the frequency shift data.

\begin{tabular}{|c|c|c|c|c|}
\multicolumn{2}{c}{ Experimental Results } & \multicolumn{2}{c|}{ MAFIA predictions } \\
\hline & $f$ & $Z / Q$ & $f$ & $Z / Q$ \\
\hline TM 110 & $265 \mathrm{MHz}$ & $12 \mathrm{ohms}$ & $264 \mathrm{MHz}$ & $9.9 \mathrm{ohms}$ \\
\hline TM 120 & $426 \mathrm{MHz}$ & $6.6 \mathrm{ohms}$ & $417 \mathrm{MHz}$ & $6.3 \mathrm{ohms}$ \\
\hline TM 130 & $652 \mathrm{MHz}$ & --- & $641 \mathrm{MHz}$ & $3.3 \mathrm{ohms}$ \\
\hline
\end{tabular}

\section{Table 1}

The test cell was also used to determine the best places to insert ferrite damping material to reduce the $Q$ 's of the modes, as mentioned before. In the initial (TOS) studies with the Rexolite insulator, ferrite pieces were placed at different radial positions along the radial line and on the outer shorting plate. These tests indicated that the positions nearest the insulator were the most effective, as might be expected.

In the second study, which involved the high dielectric constant insulator, slabs of ferrite $1 / 2$-inch thick and about $4 \times 5$ inches on a side were placed on the "back plate" behind the 

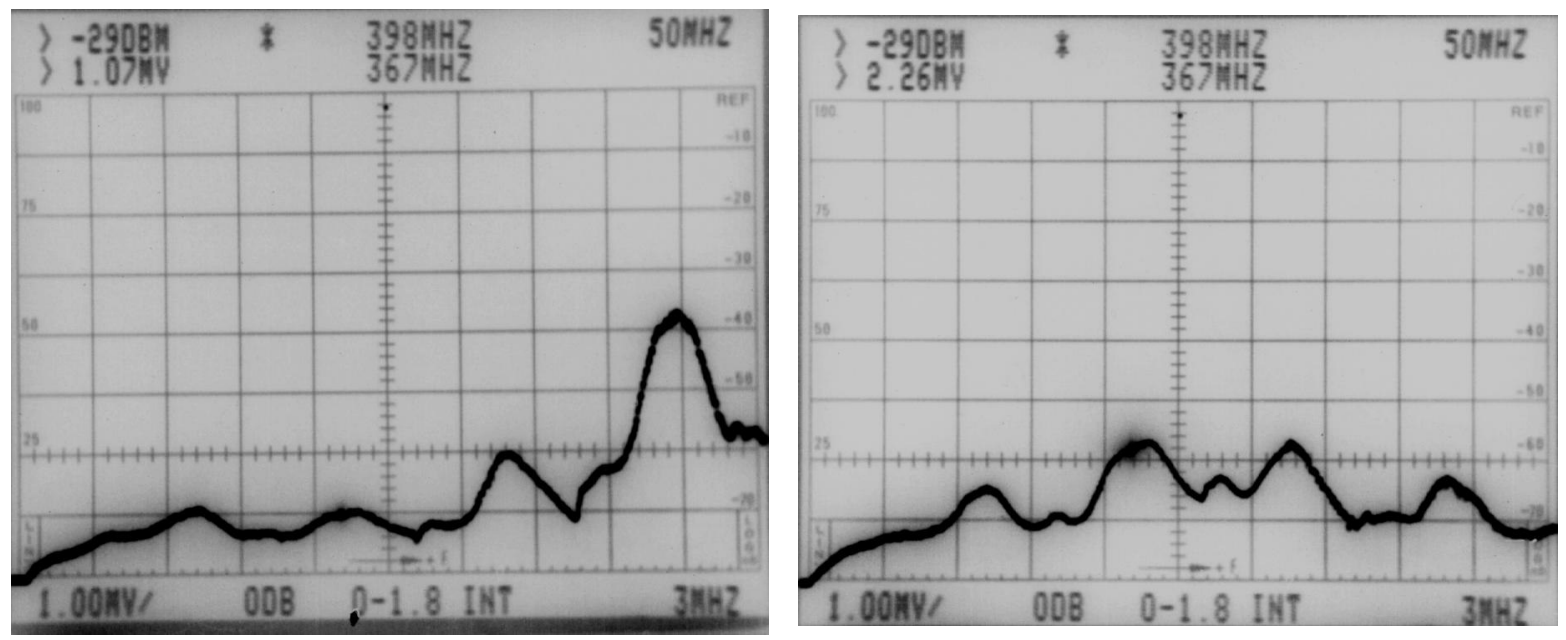

Figure 5: RF measurements showing the damping effects of ferrite. The left trace shows loop pickup data for ferrite placed behind the insulator while the right is for a configuration with ferrites placed both behind the insulator and near the acceleration gap.

insulator, as shown in Fig. 2. This ferrite dramatically lowered the $Q$ 's of all the modes, as shown in the loop response data in Fig. 5. The TM130 mode at around $600 \mathrm{MHz}$ is the most "prominent" mode remaining; its $Q$ is difficult to determine accurately, but appears to be in the range $5-8$.

When additional 1/2 inch thick ferrite pieces were placed around the outer wall of the "coax" near the gap (on the "vacuum side") as shown in Fig. 3, the response changes as shown in Fig. 5. The TM130 mode is "lowered in amplitude", while the TM110 and TM120 modes are "elevated". All modes have $Q$ 's less than 8 in this case also, but it is not clear from this data with our coupling loop excitation and detection system whether the BBU interaction would actually be mitigated significantly by the additional ferrite.

For a given mode, the peak in the resistive impedance is related to $Z / Q$ by the relation

$$
Z_{\perp r}=\frac{\omega}{c}\left(\frac{Z}{Q}\right) Q \quad \text { ohms } / \mathrm{m}
$$

With a $Q \sim 5-8$, the $Z / Q$ measurements for the modes listed in Table 1 indicate an impedance of order $330-530 \mathrm{ohms} / \mathrm{m}$. Consequently, these preliminary studies showed that the ferrite dampers were effective in reducing the mode $Q$ 's. However, they could only give a very rough indication of whether the overall impedance goals for DARHT-2 could be met. 


\subsection{Measurements on the First Prototype Cell.}

Figure 6 shows a cutaway view of the first prototype DARHT-2 accelerator cell (hereafter called "Proto-1"). This cell had actual oil-filled Metglas cores, a high dielectric constant Mycalex insulator, and fully installed pulse power drive cables and compensation resistors. For measurement purposes, a 10-inch diameter beam tube extension at least 12 inches long was mounted on the cell flange nearest to the gap. As in the previous test cell measurements, this configuration provides an accurate representation of the RF properties of the accelerator cell below the cutoff frequency of the beam tube $(690 \mathrm{MHz})$.

We found that the frequency-shift technique used with previous test cells to measure $Z / Q$ could not be used in Proto-1. An example of a frequency scan over the band 0-500 $\mathrm{MHz}$ showing the coupling between two loops inserted in the gap region is shown in Fig. 7; in the case shown, the loop coupling was between single loops on opposite sides of the gap both oriented in the radial direction. Resonant modes are clearly visible, but because the $Q^{\prime}$ s are 30 or less, placement of perturbing metal cylinders in the beam tube induce small frequency shifts which effectively are not measurable.

At this point, we began to explore a new technique for measuring the transverse impedance of heavily damped cells. The initial development of this technique and the impedance measurements on prototype cell \#1 are documented in Ref. [5]. The defining

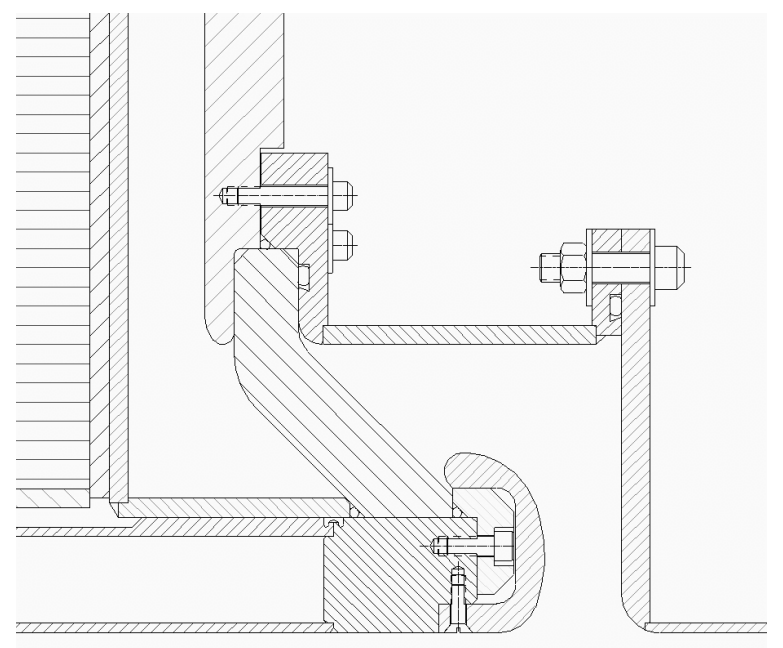

Figure 6: Diagram of the gap and insulator region of the "Proto-1" accelerator cell.

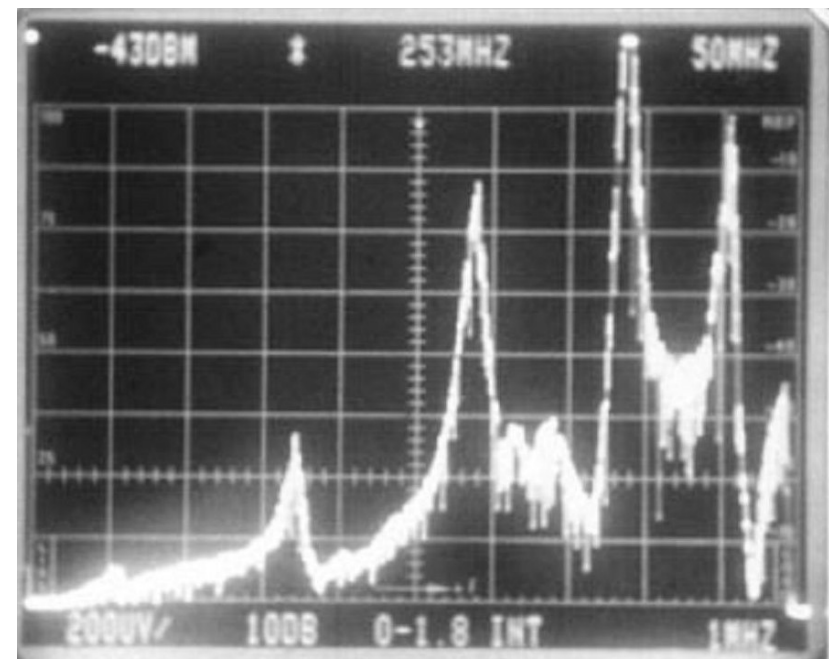

Figure 7: Loop coupling with $B_{\text {rg }}$ drive on top and

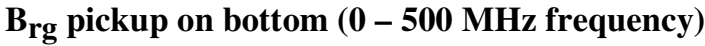
feature of this technique is the use of a twin-lead transmission line placed along the axis of the beam tube to excite the RF fields in the cell. With the twin lead terminated in a matched load, a dipolar, traveling wave is created which has the exact same electromagnetic field structure as that of a relativistic beam oscillating in the transverse direction.

The resonant modes excited in the prototype cell by this "simulated e-beam" can be clearly detected on a balanced set of radially-oriented RF-pickup loops located in the gap region as shown in Fig. 8. The main resonant frequencies ---170, 285, and $380 \mathrm{MHz}---$ are the same as in the loop-to-loop coupling experiment. (Note that the loop-to-loop coupling also indicated fairly sharp resonances around 450 and $500 \mathrm{MHz}$, but these are likely due to quadrupole or sextupole modes that are not as strongly excited by the twin- 
lead. The "purity" of the dipolar excitation, more closely simulating an oscillating e-beam on axis, is a key advantage of the twin-lead technique.)

We defer a detailed discussion of the theoretical basis of this new impedance measurement technique to Section 3 but the essential idea is illustrated in Fig. 9. In addition to the measurement of the radial rf magnetic field in the gap $\left(B_{r g}\right)$, the azimuthal $r f$ magnetic field at the beam tube wall $\left(B_{\theta v}\right)$ is also measured using the same (or identical) loops at an axial position more than a tube radius away from the gap. In both cases, a balanced set of loops in an "up-up" orientation was used, as in the test cell, to select the dipole field

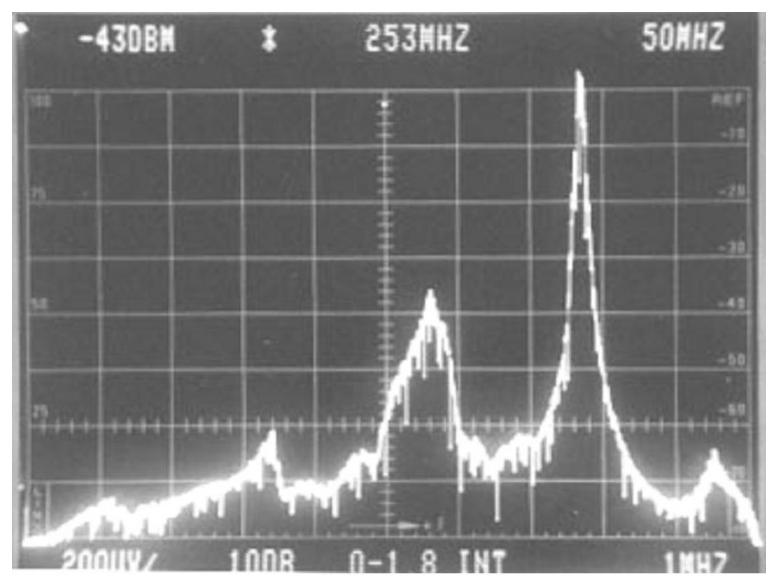

Figure 8: Resonant modes excited by a twin lead and measured by radially-oriented $\mathrm{RF}$ pickup loops components. The measurement of $B_{\theta v}$ basically calibrates the strength of the twin lead dipole excitation.
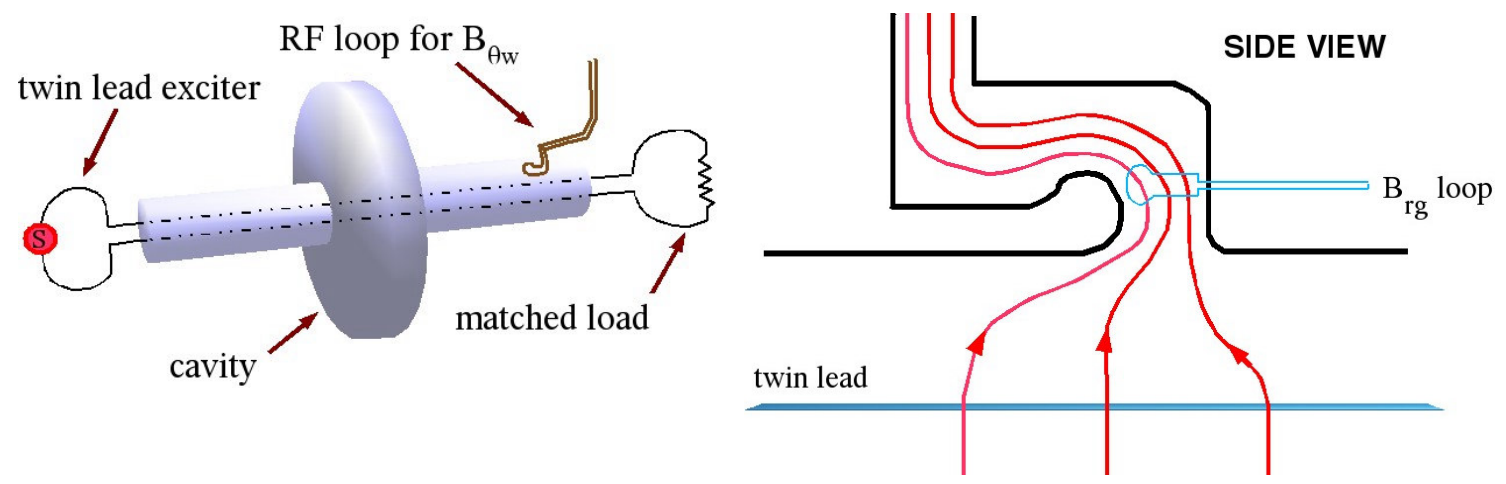

We define the quantity $\eta$ as the ratio of these two complex field amplitudes:

$$
\eta=B_{r g} / B_{\theta w}
$$

where the phase difference between the two fields must be adjusted to account for the propagation of the TEM twin lead mode from the measurement position of $B_{\theta v}$ to the gap midplane. We show in Section 3 that the full, complex transverse interaction impedance is given by

$$
Z_{\perp}=-j \sqrt{\frac{\mu_{o}}{\varepsilon_{o}}} \frac{w}{\pi b^{2}} \eta \quad \text { ohms/meter }
$$

Note that the ratio of the two RF loop signals determines $\eta$, and, importantly, this ratio is independent of both the frequency-dependent response function and the amplitude

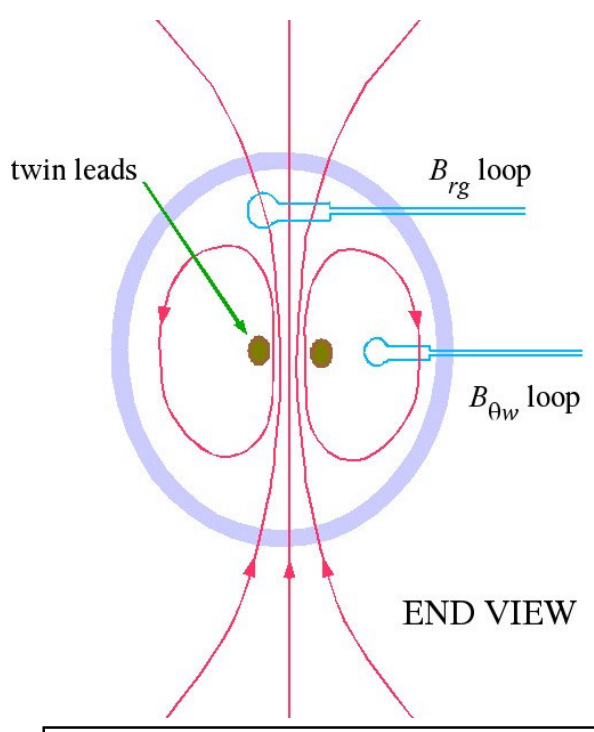

Figure 9: Illustration of twin-leadloop measurement technique calibration of the RF loops. Also, $\operatorname{Im}(\eta)=\eta_{f}$, the form factor introduced in Eq. 2.2. 
The experimental complications initially encountered in application of this technique are detailed in Ref. [5]. These complications included the need both to suppress the monopole and dipole resonances on the twinlead, and to discriminate against monopole ("common mode") RF fields on the twin-lead. As a consequence, the experimental set-up and the measurement techniques both evolved during the course of this campaign. The "final" experimental configuration that resulted from this effort involved the use of a network analyzer to measure the complex impedance (real and imaginary parts) of the final cell design. This "final" configuration is described in

Section 3, and the results are presented in Section 4. For the mode studies of the earlier prototypes described in this section, a simple sweep frequency generator and receiver were used so we could only infer the magnitude of the impedance using this technique.

Three configurations of damping ferrite were tested on Proto-1. In all cases, the ferrite material used was obtained from ERA cavities:

\section{A. No damping ferrite}

B. A damping ferrite ring near the gap on the vacuum side as shown in Fig. 10. The ring was made out of ferrite blocks, which came close to touching on the inner radius of 7.75 inches, covered $3 / 4$ inches of thickness radially and 1.5 to 2 inches, axially (beveled as shown).

C. A damping ferrite ring mounted on the back plate as shown in Fig. 10. The ring was made out of 14 blocks of $1 / 2$-inch thickness cut to fit with a gap of 0.090 inches between blocks (see Fig. 11). The radial extent of the ring was about 2 inches, with an ID of 13.6 inches. The gaps

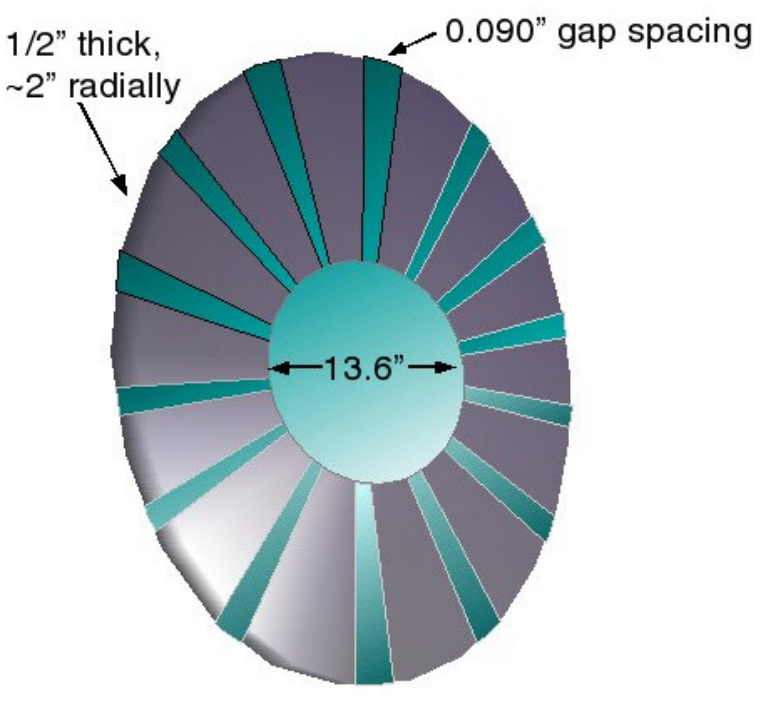

Figure 10: Drawing of "Proto-1" cell with ferrite damping ring on the oil-side back plate (to the left of the insulator) and the "vacuum-side" ferrite ring (to the right, shaded black/mauve). between the blocks are needed to avoid saturation of the ferrite from the azimuthal magnetic field of a 4-kA electron beam.

The amplitude of the $B_{r g}$ loop pickup for all three configurations is shown in Fig. 12, with the signal generator driving the twin lead swept over the range from 0 to $500 \mathrm{MHz}$. It is apparent from the data in Fig. 12 comparing configurations $\mathrm{A}, \mathrm{B}$, and $\mathrm{C}$ that the back plate ferrite is the most effective in damping the modes. Without any damping ferrite, the strongest mode in this frequency range is around $380 \mathrm{MHz}$ with a $Q$ around 30 ; ferrite in 

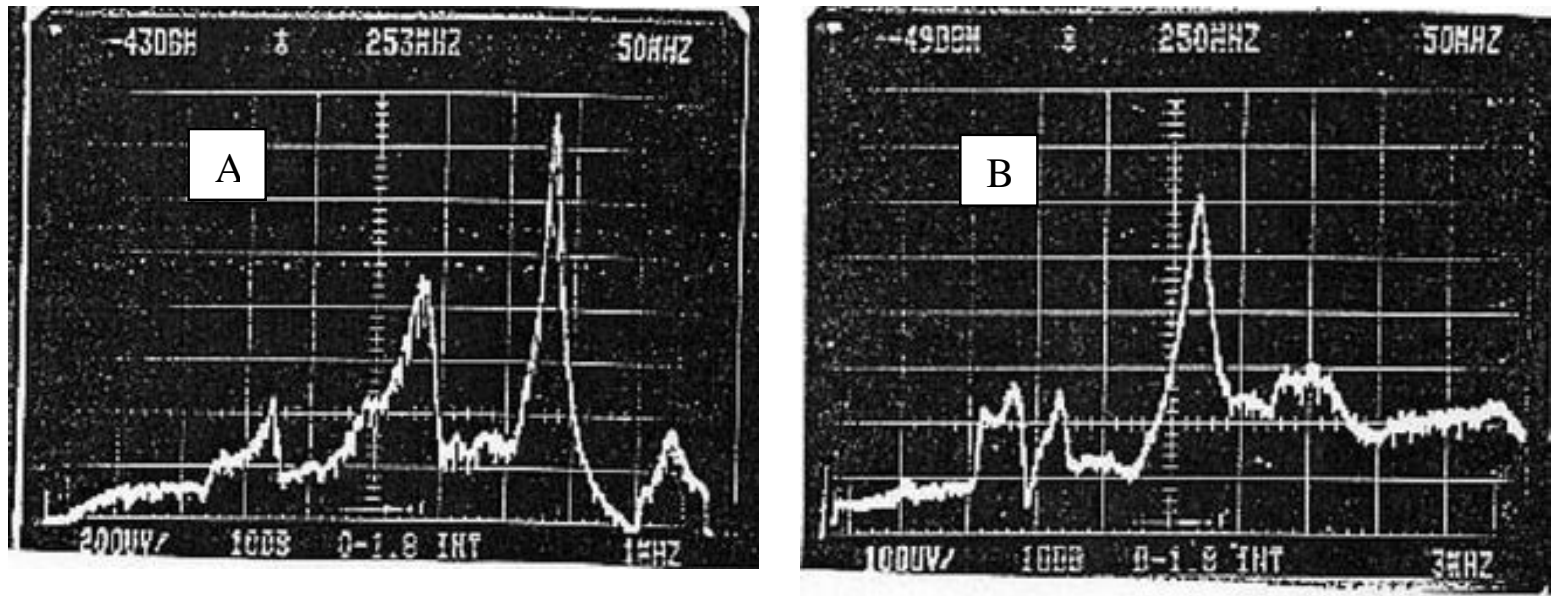

either location effectively kills this mode. The remaining mode in the $200-300 \mathrm{MHz}$ band has its frequency shifted downward when ferrite is inserted in either location. The back plate ferrite is the more effective in reducing the $Q$. Since the placement of ferrite material in the vacuum region is less desirable anyway, we concentrated exclusively on the back plate ferrite placement for the rest of the campaign.

To infer the magnitude of the impedance factor $\eta$ for configuration $\mathrm{C}$, the $B_{r g}$ loop pickup in Fig. $12 \mathrm{C}$ must be divided by the dipole $B_{6 w}$ loop pickup shown in Fig. 13. Both of these signals were taken with an identical set of balanced loops oriented in an "up-up" direction with the receiver on the same scale so the calibration is irrelevant. The $B_{6 w}$ loops were located axially one beam tube radii ( 5 inches) away from the accelerator gap. Note that the dipole twin-lead current excitation of the cell monitored by this loop varies smoothly with frequency, so the resonant features apparent in $B_{r g}$ can be used in a first approximation to determine mode $Q$ 's, etc. .

A significant geometrical correction factor must be applied to the "raw" value of $\eta$ obtained from ratios of the two loop signals, as discussed in detail in Section 3. The radial magnetic field in the gap is measured at the position indicated in Fig. 9,

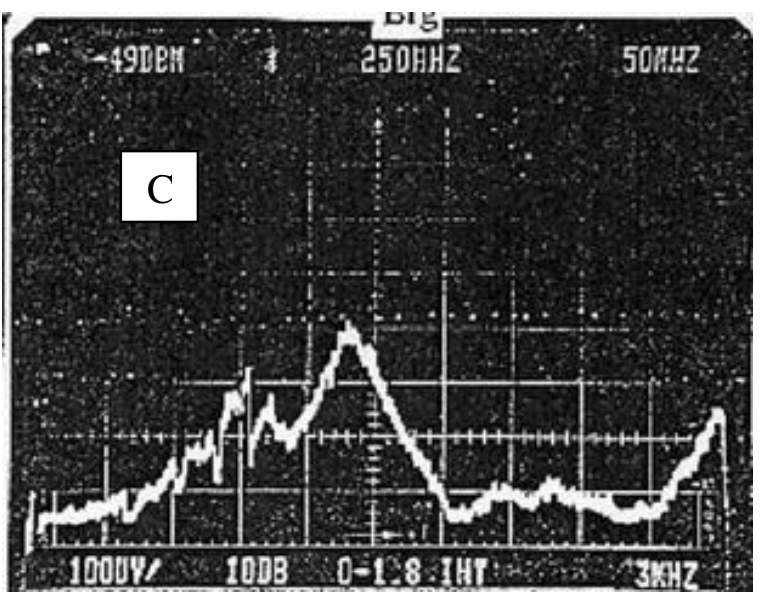

Figure 12: Mode pickup measurements for "Proto 1" cell. (A):No damping ferrite (B): Ferrite on vacuum side only (C):Ferrite on back plate (oil side) only

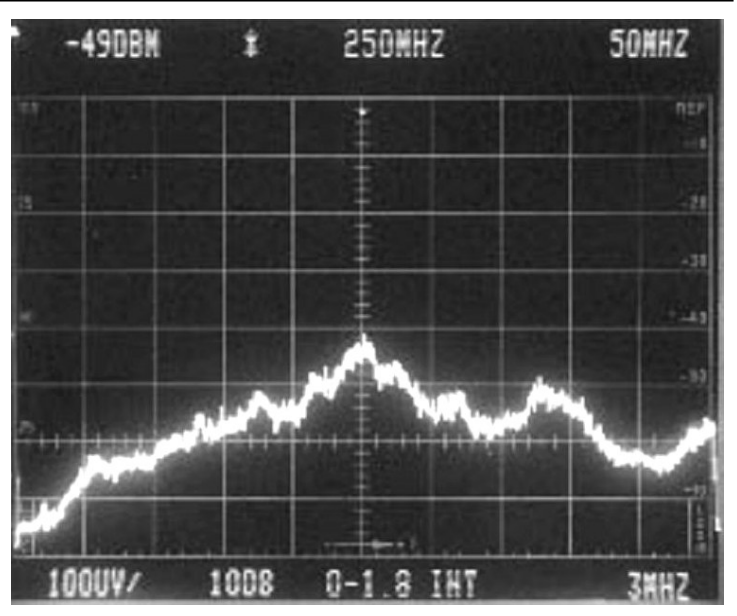

Figure 13: $\mathbf{B}_{\theta \mathrm{w}}$ loop pickup measurement $\Delta r=1.25$ inches above the beam tube radius $b=5$ inches that is the reference point in the theory. In the initial write-up of these measurements (Ref. 5), a range of correction factors was proposed depending on assumptions about the field shape. Since then, we recognized that a better estimate of the correction factor could be obtained using 
measurements of the azimuthal dipole RF field in the gap $\left(B_{\theta g}\right)$ at the same radius as $B_{r g}$ (but rotated by an angle of 90 degrees following the dipole field pattern). Defining the "shape parameter" $s$ by

$$
s=-B_{\theta g} / B_{r g}
$$

the geometrical correction factor multiplying the "raw $\eta$ " is

$$
g=1+\frac{\Delta r}{b}(1+s)
$$

A static dipole field pattern decaying radially as $1 / r^{2}$ has $s=1$, while a "monopole type decay" which scales as $1 / r$ has $s=0$. The range $1>s>0$ was used to estimate the impedance in Ref. [5]. In the "final measurement sets" described in Section 4, where a network analyzer was used to get phase as well as amplitude information, Eq. (2.7) can be used to get much more accurate corrections. With only amplitude information, however, we will use the magnitude of $s$ in Eq. 2.7 to make a "first order" estimate of the correction factor for all the cases in this Section. An additional smaller geometrical correction arises from the finite size of the loops measuring $B_{\theta w}$; this results in an additional multiplying factor of 1.088 applied to the "raw" $\eta$.

From loop measurements over the full frequency range up to the cutoff frequency of the beam tube at $690 \mathrm{MHz}$, we deduced the values of $Q$, "raw" $\eta$, and magnitude of $s$ for the two dominant modes as presented in Table 2 (located at the end of Section 2.7). The magnitude of $\eta$ and magnitude of the transverse impedance obtained using the estimated geometrical correction factors are also given in Table 2. Error bars and uncertainties are not explicitly stated, but a range of order $+/-10-20 \%$ should clearly be understood to apply to these peak impedance values.

We also emphasize that the real part of the impedance is the quantity that enters into BBU growth rate calculations. As will be illustrated in Section 4, the imaginary part of the impedance is not negligible even at resonance in these low $Q$ situations, so the magnitude of the impedance is only a rough guide to the BBU growth (a point not fully appreciated when Ref. [5] was written). 


\subsection{Measurements on Prototype Cell \#3.}

The gap region of the third prototype cell is shown in Fig. 14. This cell was similar to the first prototype, but a number of changes were made as the mechanical design matured. The axial extent of the gap was shortened, in a constant quest to keep the accelerator length as short as possible. The insulator now made an angle of about 30 degrees to the axis, vs. about 45 degrees in Proto-1. The ferrite on the back plate was roughly comparable in both cases, although the total volume of ferrite was slightly less in Proto-3 (by 12\%) and it had more plates and larger gaps spacing than Proto-1.

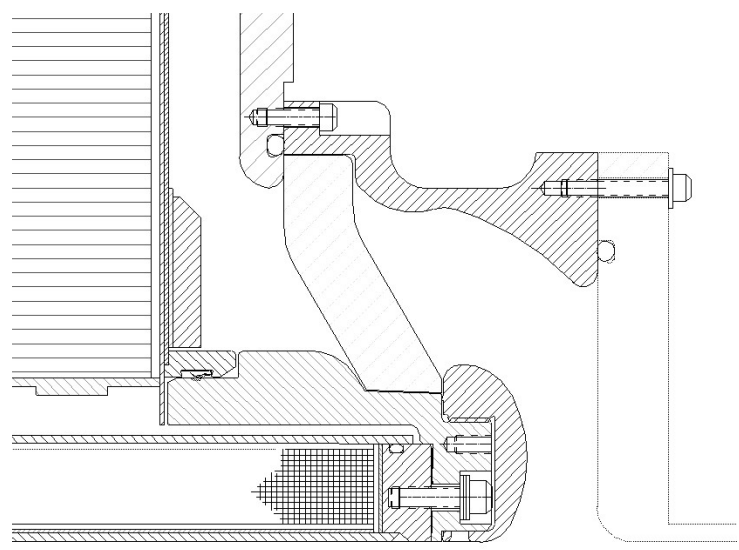

Figure 14: Prototype 3 gap region

A significant improvement in the experimental setup was made with the use of a broadband balun to connect the twin-lead to the generator. This ensured much purer balanced dipolar excitation of the twin-lead with even smoother frequency dependence.

Frequency scans over 0 to $1 \mathrm{GHz}$ of the $B_{r g}, B_{\theta w}, B_{\theta g}$ loop signals are presented in Fig. 15. It is immediately apparent even from the raw data that the modes in Proto-3 have much higher $Q$ 's and higher impedances than Proto-1. (Note: the sharp spike at $690 \mathrm{MHz}$ that is very prominent on the $B_{\theta}$ scan is from passing through the cutoff frequency of the beam tube). The values derived from this data using the same procedures and approximations as in Section 2.3 are given in Table 2 in Section 2.7.

The dramatic increase in the $Q$ of the high frequency mode was especially troubling. The central question of course is why such "minor" changes in the configuration lead to such a result. This prompted the next phase of the campaign described in the following Section.

\subsection{Modeling Studies and Optimization of the Damping Ferrite}

The main variables at our disposal to minimize the $Q$ 's and the impedance were discussed in Section 2.1. At this stage of the project, the main features of the mechanical design and the insulator were considered "frozen". Shaping of the outer electrode in the vacuum region was one feature easy to modify, so variations in this area were considered and modeled in the codes. The other was the shape and
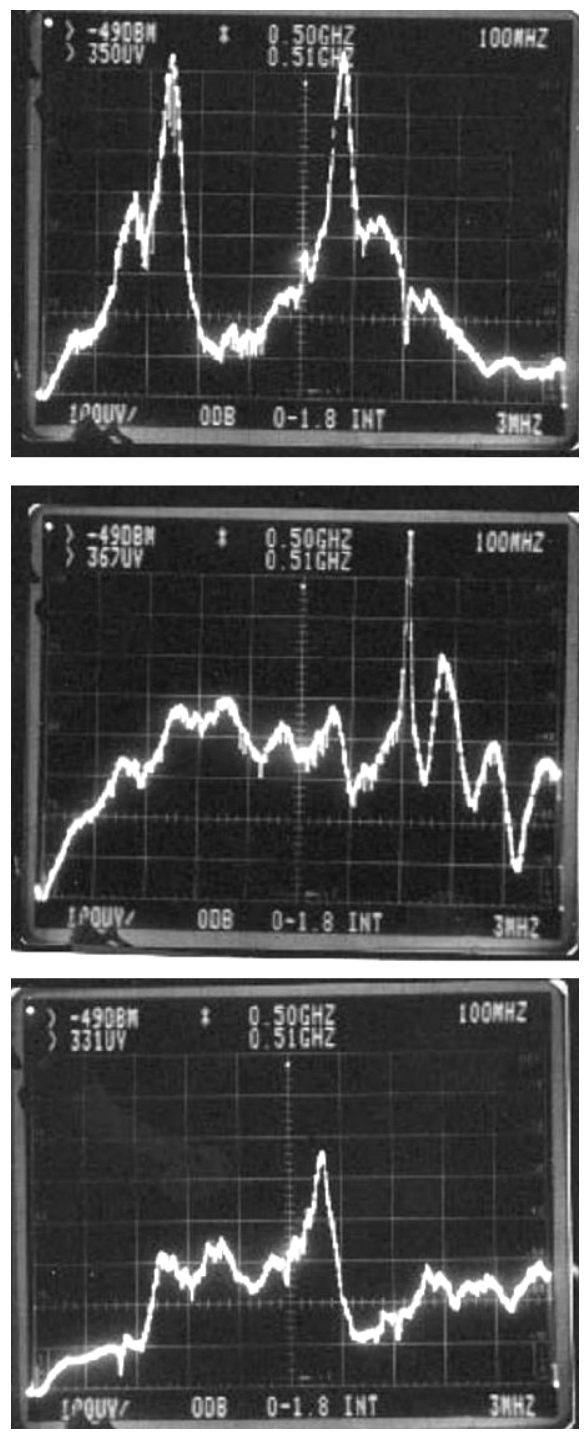

Figure 15: From top to bottom:

$\mathbf{B}_{\mathbf{r g}}, \mathbf{B}_{\theta \mathbf{w}}$, and $\mathbf{B}_{\theta \mathrm{g}}$ pickup signals in the 0 to $1 \mathrm{GHz}$ frequency range for the Proto-3 cell. 
thickness of the ferrite on the back plate.

2.5.1 Physical insights into optimization of the ferrite thickness.

Ferrite material is a common choice for electromagnet absorber applications in the VHF and UHF frequency bands. Above $30-50 \mathrm{MHz}$ or so, in ferrites appropriate for this application the imaginary part of the permeability is much larger than the real part of the permeability, and it drops off with frequency like

$$
\mu^{\prime \prime} \approx M\left(\frac{100 M H z}{f}\right)
$$

Deford and Kamin [6] quote a value $M \sim 90$ in thin sample measurements of ATA/ETA ferrite, and a permittivity $\varepsilon=13$. Catalog data for an appropriate TDK absorber appropriate for the frequency band of the main BBU modes in DARHT-2 (IB-011) has $M \sim 75$ and $\varepsilon=11$. Measurements by Burke and Nelson of the N23 ferrite tiles from CMI technology that were ultimately installed in (most of) the DARHT-2 cells are similar to the TDK data, namely $M \sim 80$, and $\varepsilon=14$.

The propagation constant of a plane EM wave in the ferrite in this frequency range is given by

$$
\begin{aligned}
& \gamma=(1+j) \alpha \\
& \alpha=\frac{\omega}{c} \sqrt{\varepsilon \mu^{\prime \prime} / 2}
\end{aligned}
$$

The exponential decay length is therefore about $2 \mathrm{~cm}$ at $100 \mathrm{MHz}$ and varies as the inverse square root of frequency. One key attractive feature of ferrite as an absorber that this illustrates is that relatively thin samples (e.g., a thickness much less than a free space wavelength) can have appreciable losses.

With a slab much thicker than the decay length, the wave impedance for normal incidence is

$$
Z=E_{x} / H_{y}=\omega \mu_{o} \mu " / \gamma
$$

The magnitude of the wave impedance in the ferrite in the several-hundred-MHz band is comparable to the free space wave impedance, since the ratio $\sqrt{\mu " / \varepsilon}$ is of order unity. Since the reactive component of the impedance of the thick slab is approximately equal to the real part of the impedance, however, there are still appreciable reflections. Using the TDK data, the plane wave reflection coefficient of a thick slab is about 0.6 at $200 \mathrm{MHz}$. 
Catalog data on VHF/UHF absorbers (from TDK, for example) indicate that a deep minimum in the reflection coefficient over a limited frequency band can be obtained with relatively thin ferrite slabs mounted on a conducting plate. The "trick" is to pick a ferrite thickness so the decaying wave reflected off of the metal plate tends to cancel the reactive part of the incident wave impedance. To illustrate the dependence on thickness, we present a calculation

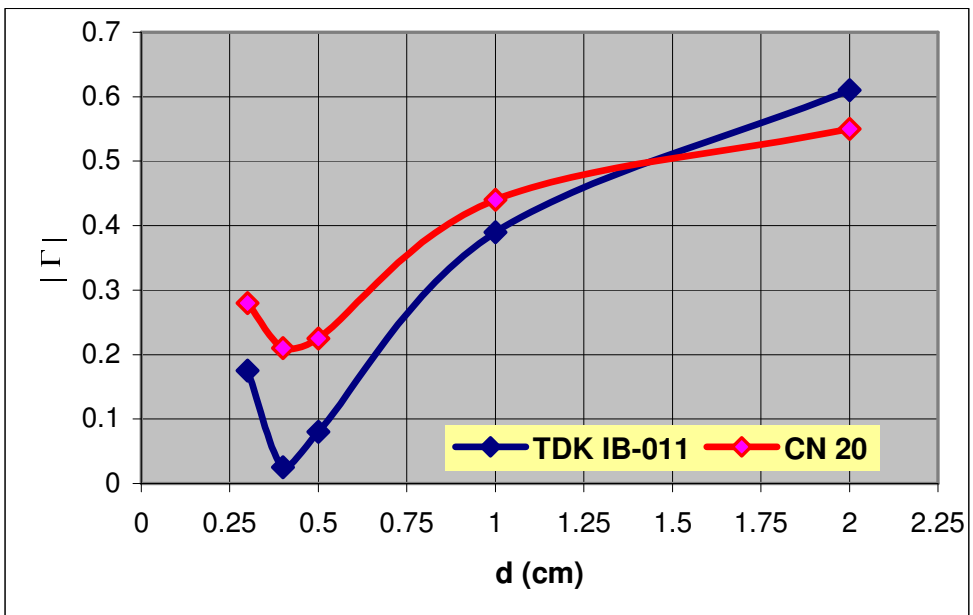

Figure 16: Reflection coefficient at $200 \mathrm{MHz}$ vs. thickness of ferrite slab in oil. of the wave reflection coefficient with a normal incident plane wave on an oil-ferrite interface in Fig. 16. The calculation used the TDK ferrite parameters, a frequency of $200 \mathrm{MHz}$, and an oil dielectric constant of 2.3. The result is quite remarkable: a deep null in the reflection at a thickness of $4 \mathrm{~mm}$, where the one-way phase shift through the slab is about 14 degrees and the one-way attenuation is about $1 / 4$ of an e-fold.

Fig. 16 also shows a second curve representing measurements of $\mathrm{CN} 20$ ferrite by Walter Barry at LBNL. In this case, the $\operatorname{Re}(\mu)=15$ is about half the value of $\operatorname{Im}(\mu)$ at $200 \mathrm{MHz}$ and it does not produce as deep a minimum as does the TDK material.

Fig. 16 illustrates that significant reductions in wave reflection compared to a thick slab can occur over a relatively broad range of thickness (i.e., it is not "sharply tuned" to a given frequency). The frequency bandwidth for minimal wave reflection with a given thickness is also quite broad; typical catalog specs on specific ferrite tiles quote reflections below $10 \%(20 \mathrm{~dB}$ ) over 30 to $400 \mathrm{MHz}$ (or 100 to $600 \mathrm{MHz}$ for a slightly thinner tile). Selection of an appropriate tile thickness should therefore significantly enhance the wave absorption over the full frequency band of interest in BBU mode suppression.

A serious caveat in applying these ideas directly to the DARHT cell geometry is the obvious fact that the RF fields in the cavity are not plane waves at normal incidence to the ferrite slabs. However, the fields inside the ferrite will still be reasonably well approximated by plane waves "propagating" normal to the interface because of the large value of both $\mu$ and $\varepsilon$; this situation is somewhat similar to the field penetration in a good conductor. With the proper thickness choice, the surface impedance at the ferrite-oil interface will still be mainly resistive and close to the characteristic wave impedance (in oil). So picking the ferrite tile thickness on the basis of minimizing the reflectivity of normally incident plane waves should be a good starting point for more detailed computational studies. 


\subsubsection{AMOS modeling studies.}

As part of the campaign to reduce the cell impedance to an acceptable level, an extensive numerical simulation effort was undertaken by S. Nelson at LLNL using the AMOS code. AMOS is a $2.5 \mathrm{D}$, finite difference, time-domain electromagnetic code that computes the spatially resolved temporal response to a dipole current excitation. The code can model vacuum, dielectric, and magnetoactive material regions. Normally, the temporal field information is then Fourier transformed to determine the frequency resolved $Z_{\perp}$ and $Q$ 's of the system.

Some AMOS results for DARHT-II have

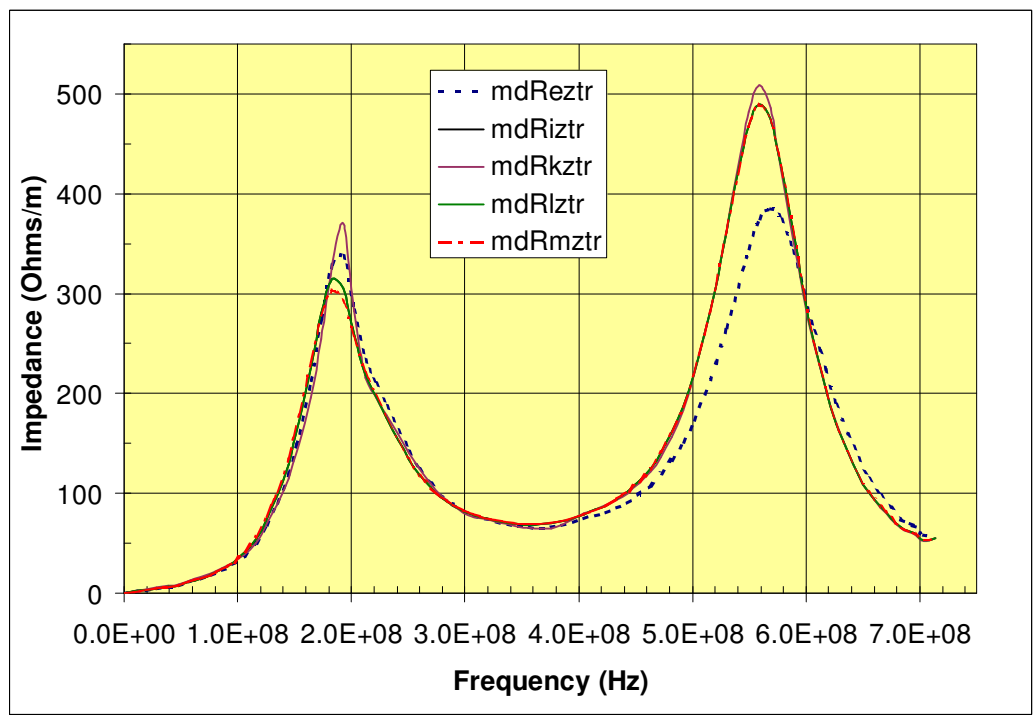

Figure 17: AMOS impedance predictions for the standard cell as a function of ferrite damper length. been reported elsewhere (see Ref. $[7,8]$ ) and here we will only summarize key findings.

First, the initial design (e.g. Proto-I) showed two major resonance peaks with $Z_{\perp}$ in the range of 600-700 ohms/meter and $Q$ 's above 10. As was discussed in previous sections, a key method to reduce the impedances to more acceptable values was to put ferritedamping material in the oil region immediately upstream of the insulator. AMOS was used to examine the sensitivity of the predicted impedance to the radial length of ferrite dampers. As can be seen in Fig. 17, the $Z_{\perp}$ corresponding to the lower frequency resonance around $200 \mathrm{MHz}$ slowly decreases from 400 to $300 \mathrm{ohms} / \mathrm{m}$ as the ferrite length varies from 10 (run mdRkztr) to $15 \mathrm{~cm}$ (run mdReztr).

We were also interested in the sensitivity of the impedance to the angle of the corner reflector in the anode region of the acceleration gap. Fig. 18 displays AMOS calculations of this sensitivity. One sees that the impedance of the $200 \mathrm{MHz}$ resonance is nearly constant but $Z_{\perp}$ for the higher frequency resonance just below $600 \mathrm{MHz}$ drops from 475 to $375 \mathrm{ohms} / \mathrm{m}$ as the angle drops from 60 to 30 degrees. A zero degree reflector is parallel to the centerline axis while one at 90 degrees is perpendicular (i.e., in the radial direction). The angle in the as-built DARHT-2 cell is approximately 45 degrees (see Fig. 19) but has some element of curviness. These results suggest that a fairly

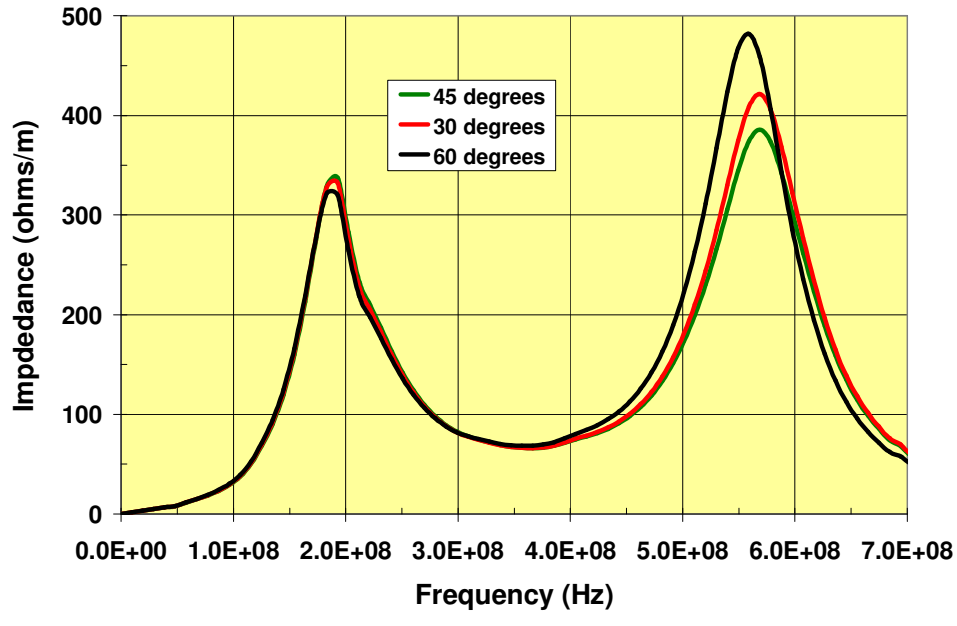

Figure 18: AMOS impedance predictions for the standard cell as a function of anode corner reflector angle. broad range of the actual corner reflector angle is acceptable. 
One key thing to note from the calculations presented in these figures is that the low frequency resonance has a simple single peak structure. This is in contrast to the experimental measurements (see Sections 2.6 and 4), which show a double peak. To this date it remains unclear as to the cause of the double peak.

\subsection{Final Design of the Standard Cell, and Initial Magnitude of $\mathbf{Z}$ Measurements}

The final design configuration shown in Fig. 19 was chosen on the basis of the ferrite thickness optimization considerations and modeling studies discussed in Section 2.5. The ferrite tiles are the most pronounced difference between this configuration and Proto-3 (Fig. 14). In the final configuration the ferrite tiles are 5.5-mm thick, about half of the thickness of the Proto-3 tiles. Being much thinner, they could also be extended a greater radial distance outwards as shown. Figure 20 shows a drawing of the ferrite tile ring in the standard cell. In the final design the electrode shape in front of the insulator was also angled down to improve the coupling of RF energy through the insulator, as suggested by the AMOS code studies discussed in the previous section. The insulator itself was not changed, as already mentioned.

To meet the project delivery time schedule, the first 8 standard cells used immediately available catalogue ferrite tiles from TDK (IB-011), not quite as tall as the final design, and an older finger stock geometry (see Fig. 21). The RF properties of these cells should be very close to those measured for the final configuration, but this difference is noted here for record keeping purposes.

To test the final design, Proto- 1 was modified to have the correct electrode shape, insulator, and ferrite tile as shown in Fig. 22. The experimental set up was identical to the one used in the Proto-3 impedance measurements.

Frequency scans over 0 to $1 \mathrm{GHz}$ of the $B_{r g}, B_{\theta w}, B_{\theta g}$ loop signals are presented in Fig. 23. Comparing the $B_{r g}$ scans of the final design with Proto- 3 we note a reduction by more than a factor of 2 in the Q's of both of the main modes. An estimate of the magnitude of the impedance

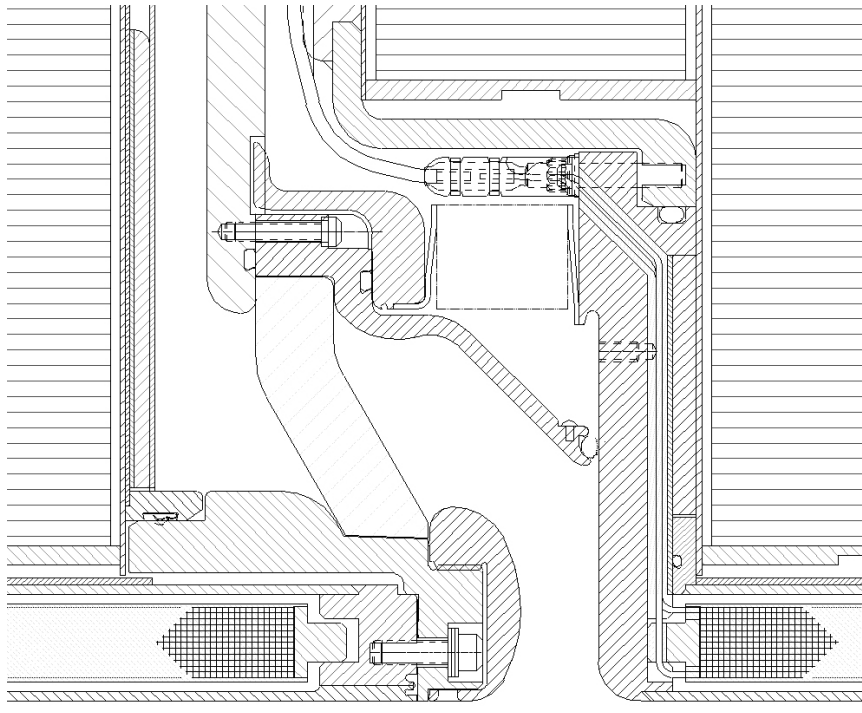

Figure 19: Final standard cell design.

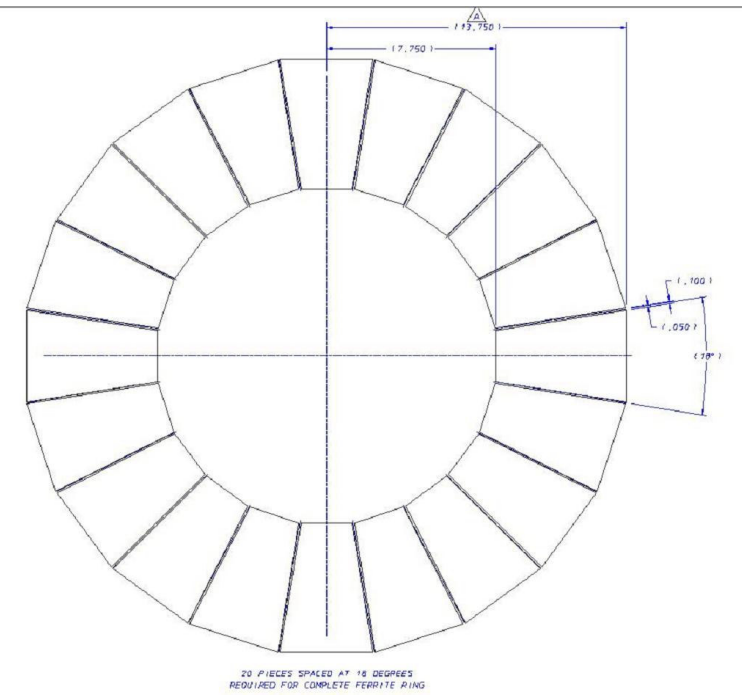

Figure 20: Drawing of ferrite damping tiles showing azimuthal layout. from this loop data was made following the same procedures and assumptions as in Section 2.3. This estimate and the other mode parameters derived from the loop data are given in the third line in Table 2 at the end of Sec. 2.7. 
Note that in the final design, and to a lesser extent in Proto-3, the $B_{r g}$ scans exhibit a double peak (at 160 $\mathrm{MHz}$ and $240 \mathrm{MHz}$ ) around the lower resonance (mode splitting?). We did not include the lower frequency peak in Table 2, however, for the final design an estimate of the magnitude of $\eta$ and $Z_{\perp}$ indicates that the lower frequency peak $(160 \mathrm{MHz})$ is about the same height as the upper frequency $(240 \mathrm{MHz})$. This doublehumped character around $200 \mathrm{MHz}$ will be discussed in detail in Section 4, where we present the results of phase as well as amplitude measurements.

\subsection{Concluding Comments}

The discussion in Section 2 focused heavily on the steps we went through to arrive at the final design of the RF features in the accelerating cells. We have only briefly alluded to our struggles to develop a useful technique for measuring the cell impedance. In particular, we did not discuss at all the efforts (see Ref. [9]) to measure the impedance using the TSD technique that had been applied before on the DARHT-1 cell. The large bore and low mode frequencies made the TSD technique much more difficult to apply in DARHT-2 cell measurements, and the mode structure so clearly evident on the loops was barely discernable in the TSD data (which also required heavy filtering of fixture resonances).

The "twin-lead-loop hybrid" technique was developed and refined throughout this campaign, and it proved to be a very useful technique. Towards the end of the campaign, we had refined the twin-lead matching and excitation system to the point where a very "pure" traveling wave dipole wall current was induced by the

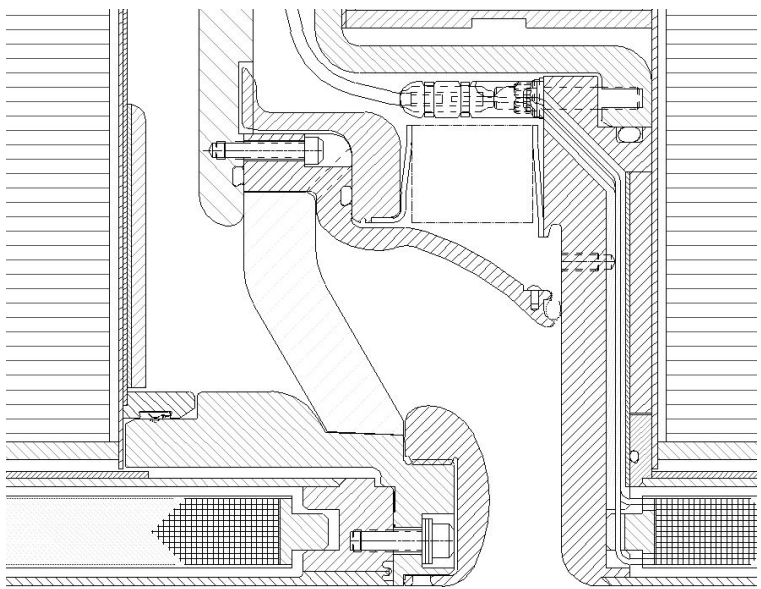

Figure 21: As built geometry of the first 8 standard cells delivered to LANL.

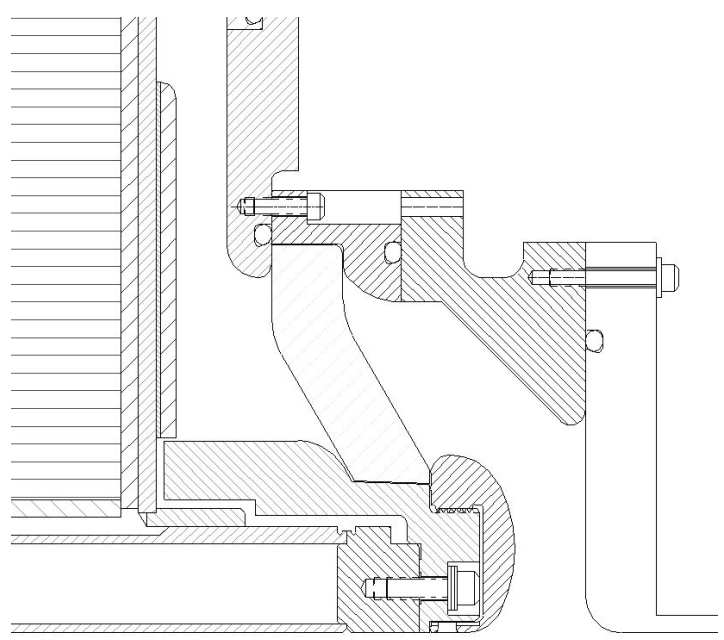

Figure 22: Modified Proto-1 cell geometry for RF measurements. twin-lead outside the gap (measured by $B_{\theta_{w}}$ ) that varied smoothly and monotonically with frequency (see Fig. 23). With this kind of "smooth" excitation even the "raw" (unprocessed) $B_{r g}$ frequency scans are sufficient to determine the frequencies and the $Q$ 's of the modes. Smooth variation of the drive also makes it easy to do a quick estimate of the impedance from the loop signal ratios. As an additional test of the quality of the mode excitation, we turned one of the $B_{6 w}$ loops around (to an up-down orientation) to pick up the monopole component of wall current. We found that the monopole wall current to be less than 10-20\% of the dipole wall current over the full frequency band below cutoff in the last two prototypes; this is in contrast to the earlier measurements reported in Ref. [5]. The use of the balun to provide a well-balanced dipole excitation of the twin-lead was a major factor in this improvement. 
To some extent, the data presented in Table 2 below suggests that the final design had barely recovered the performance we obtained initially with the more slanted insulator in Proto-1. While this may have some validity, the quality of the data in the initial tests makes it dangerous to draw firm conclusions. In addition, in very low $Q$ situations like these, the magnitude of the impedance is not the full story. Even at the peaks of the magnitude of $Z_{\perp}$, the reactive part of the impedance can be appreciable. With phase information from a network analyzer, as we will see in Section 4, the peak resistive part of the impedance turns out to be $20-25 \%$ less than the peaks in the magnitude shown in Table 2.
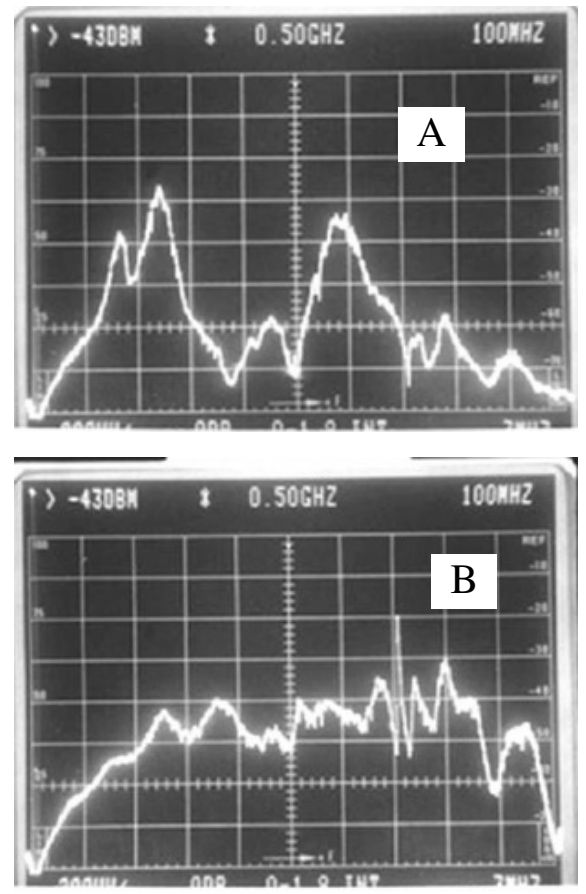

Figure 23: Frequency scans from 0 to 1 $\mathrm{GHz}$ of the $B_{r g}$ (A): $B_{\theta w}(\mathrm{~B})$ and $B_{\theta g}$ (C) loop signals for the Proto-3 configuration.

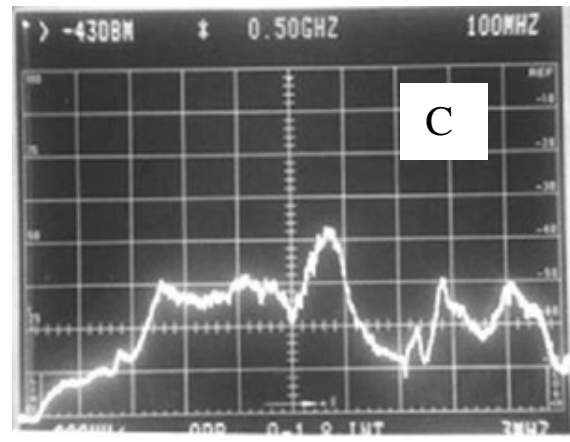

\begin{tabular}{|c|c|c|c|c|c|c|}
\hline & $\begin{array}{c}\text { Mode freq } \\
\text { (MHz) }\end{array}$ & $Q^{\prime}$ 's & $\begin{array}{c}\eta \\
\text { (loop ratio) }\end{array}$ & ISI & $\begin{array}{c}\eta \\
\text { (corrected) }\end{array}$ & $\begin{array}{c}\left|Z_{\perp}\right| \\
\text { (ohms/m) }\end{array}$ \\
\hline $\begin{array}{c}\text { Proto-1 } \\
\text { (config. C) }\end{array}$ & 230 & 5 & 1.4 & 0.35 & 2 & 380 \\
\hline Proto-3 & 250 & 6 & 1.8 & 0.35 & 2.6 & 500 \\
\hline $\begin{array}{c}\text { "Final" } \\
\text { Prototype }\end{array}$ & 240 & 3 & 1.35 & 0.5 & 2 & 380 \\
\hline
\end{tabular}

Table 2: Summary of mode parameters for the three prototype cell configurations 


\section{Twin-lead-loop Measurement Technique}

In the previous section, we briefly outlined the essence of the twin lead loop measurement technique, and how it evolved throughout the campaign testing a series of prototype cell configurations. Here our aim is to describe the technique in detail as it was finally applied measuring the impedance of the production standard cell and injector cell configurations.

The theoretical basis of the approach is presented in Section 3.1. A derivation of geometrical correction factors useful in its application is covered in 3.2, and limitations in the twin-lead spacing as a function of the impedance being measured is described in 3.3. More details on the experimental configuration used for the measurements of the production cells are given in 3.4.

\subsection{Theoretical Basis of the Measurement}

A highly relativistic electron beam of current I oscillating transversely with amplitude $\Delta_{x}$ in the $x$ direction inside a conducting beam tube of radius $b$ induces a dipole surface current on the tube wall equal to

$$
K_{z}=-\frac{I \Delta x}{\pi b^{2}} \cos \theta=K_{z o} \cos \theta
$$

The beam also induces a dipole surface charge $K_{z} / c$ on the tube wall. As is well known, the collective forces from the surface charge (attractive) and the surface current (repulsive) cancel inside a smooth conducting tube for a highly relativistic beam. When the beam passes through an accelerating gap region, the dipole surface current flows radially outward into the accelerator cell, destroying this force balance. We consider only frequencies below the cutoff for propagation in the beam tube $\left(\omega<\omega_{c o}=1.84 c / b\right)$ where the dipole modes of the cavity excited by the beam oscillation lead to localized deflection forces on the beam.

To describe the cavity excitation and the fields that produce a net transverse force, we use the principle of superposition (see Fig. 24 and Ref. [10]). We first place ideal surface current and surface charge sources at radius $b$ across the gap equal to those induced in a smooth conducting tube, as given above. The net force from these sources and the surface current and charge on the beam tube outside the gap region will sum to zero, obviously, since the fields are

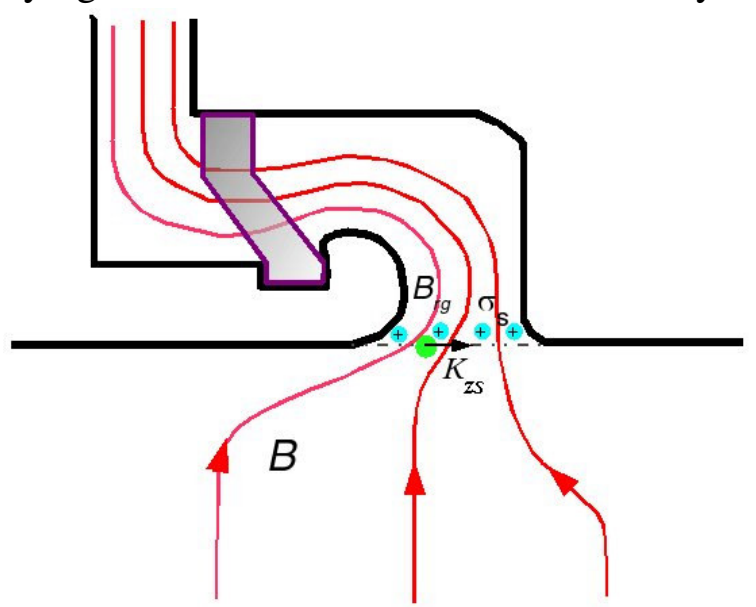

Figure 24: Equivalent surface current and charge place across the acceleration gap. Using the principle of superposition, the fields from these sources are the ones that create a net transverse force on the beam electrons. 
the same as in a smooth tube without the gap. Then, we must add surface current and surface charge sources across the gap of the opposite sign to return to the actual situation. The fields created by these localized "negative" surface current and surface charge sources across the gap (the "cavity fields") give the net force on the beam.

To determine the net transverse impulse imparted to the electrons passing through the gap region of one cell, we need to integrate the transverse electromagnetic force $E+v \times B$ along the electron trajectory in $z$ (assumed to be at a constant displacement from the axis through the gap region). For highly relativistic electrons, it can be proven that this integral is independent of radial position anywhere inside the beam tube. Using this theorem, we can relate the change in transverse momentum to the radial magnetic field and azimuthal electric field at the gap (i.e., at the beam tube radius). With a gap width much less than the beam tube radius and the wavelength, it is physically obvious that the integral of the azimuthal electric field will be very small since it is shorted out on both sides of the gap. With this assumption, the change in transverse momentum is given by

$$
\delta p_{x}=-e \int B_{r}(r=b, \theta=\pi / 2) d z
$$

The radial magnetic field at the gap is of course directly proportional to the strength of the oscillating beam's dipole moment, or equivalently the surface current source $K_{z 0}$ (and it varies as $\sin \theta$ ). The electromagnetic response of the cell itself can therefore be incorporated into a dimensionless, complex, frequency-dependent parameter defined by

$$
\eta=-\frac{1}{w} \int B_{r}(b) d z / \mu_{0} K_{z 0}=-<B_{r}(b)>/ \mu_{0} K_{z 0}
$$

Here $B_{r}(b)$ is shorthand notation for the radial field at $r=b$ and $\theta=\pi / 2$ in Eq. 3.2, and the brackets $<>$ signifies an averaging in $z$ over the gap. Introducing the definition of transverse interaction impedance

$$
\delta p_{x}=-j \frac{e}{c} Z_{\perp} I \Delta_{x}
$$

into Eq. 3.2, and using Equations 3.1 and 3.3, we obtain

$$
Z_{\perp}=-j \sqrt{\frac{\mu_{o}}{\varepsilon_{o}}} \frac{w}{\pi b^{2}} \eta
$$

with $\eta$ defined by Eq. 3.3.

It is interesting to note that a similar form for the peak value of the resistive part of the transverse impedance was introduced many years ago (see Section 2.1, Eq. 2.2). At that time $\eta$ was considered to be only a convenient figure of merit, a dimensionless "form factor" that could be reduce to of order unity in a well-damped induction cell. The derivation here provides a physical interpretation of this form factor in terms of the fields and equivalent source currents. 
This discussion carries over directly to the measurement of the transverse impedance using the twin lead excitation. The role of the twin lead is to induce a surface current in the beam tube of the same form as Eq. 3.1, except that $\Delta_{x}$ is now the spacing of the wires and $\pm I$ is the current in each wire. To accurately simulate the beam, the wave launched on the twin lead should be a pure forward (or backward) traveling wave; that is, reflections from the interaction with the gap should be minimal. As discussed in Section 3.3, this will set an upper bound on the spacing of the wires to measure a given cell impedance and mode $Q$.

To determine $\eta$ experimentally, we note that with a pure traveling wave set up by the twin lead, the measurement of dipole $B_{\theta}$ at the wall some distance axially from the accelerator gap provides a direct measure of the magnitude of the "source current" $K_{z O}$ in Eq. 3.3 (see Fig. 9). The phase must of course be adjusted. If the measurement of "surface current on the tube wall", equivalently

$$
B_{\theta v}=B_{\theta}(r=b, \theta=0)
$$

is made at an axial position $z_{0}$ away from the gap on the generator side, then in Eq. 3.3 we use

$$
\mu_{0} K_{z o}=-B_{\theta v} \exp \left(-j \omega z_{o} / c\right)
$$

The distance from the gap must be large enough for the "cavity fields" (the fields created by the equivalent "negative" surface current and surface charge across the gap discussed above) to die out. Below cutoff, the lowest mode in the tube (first positive zero of $\left.J_{1}(\omega b / c)\right)$ corresponds to an axial decay of the cavity fields as

$$
\sim \exp (-1.84 z / b)
$$

In the final round of measurements presented in Section 4, the wall loop was located at $z_{0}=3 b$ where the residual "cavity fields" should be down to less than $0.4 \%$ of their value near the gap. In all the earlier experiments reported in Section 2, the $B_{\theta_{v}}$ loop was at $z \sim b$ so larger error bars should be used in that data in relating the loop signal to $K_{z o}$.

This impedance formulation and the measurement technique are based on the assumption that the transverse force on the electrons from the azimuthal electric field can be ignored compared to the force from the radial magnetic field. It can be shown that this assumption is valid as long as the gap width is small compared to the tube radius (and wavelength). With wider gap widths, the so-called "trapped modes" near cutoff could begin to play a role (Ref. [11]). The source for these "trapped modes" is the equivalent surface charge across the gap in Fig. 24. A simple analytic estimate of the trapped mode $Z / Q$ of a radial line shows that, although the mode exists in principle for any value of $w / b$, for small $w / b$ it scales as $(w / b)^{6}$ and is completely insignificant for DARHT-2 parameters (Ref. [12]). 


\subsection{Geometrical Correction Factors for Rounded Gaps and Finite Loop Size}

The actual gap has rounded corners, as shown for example in Fig.24. The RF loop measuring $B_{r}$ in the gap was mounted at the radius indicated in the side view of Fig.9, where the gap was a minimum ( $w=1$ '), and the loop extended axially across almost all the gap. In all the DARHT cells, this loop position corresponds to $r_{p}=b+\Delta r$, where $\Delta r=5 / 4 "$ (i.e., a $25 \%$ larger radius than the beam tube in the standard cell).

We now derive a radial correction factor to more accurately relate this loop signal to the desired integral of the radial magnetic field at $r=b$ that enters in Eq. 3.3. Since div $B=0$,

$$
\frac{1}{r} \frac{\dot{o}}{\dot{o} r}\left(r B_{r}\right)+\frac{1}{r} \frac{\bar{\partial} B_{\theta}}{\dot{\partial} \theta}+\frac{\bar{o} B_{z}}{\bar{\partial} z}=0
$$

At any radius $r \geq b$, we define

$$
<B_{r}(r)>=\frac{1}{w} \int B_{r}(r, z) d z
$$

where the integral in $z$ extends from one side of the gap to the other (note that $\mathrm{w}$ is always defined as the minimum gap width). If we perform a similar integral over $z$ (at fixed $r$ ) to all the terms in Eq. 3.9, since $B_{z}=0$ at the ends of the integral (i.e., just inside the metal electrodes), we find

$$
\frac{\bar{o}}{\bar{o} r}\left(r<B_{r}(r)>\right)=<B_{\theta}(r)>
$$

where the dependence $\sin \theta$ for $B_{r}$ and $\cos \theta$ for $B_{\theta}$ is implicit. This relation provides the basis for a linear interpolation of $\left\langle B_{r}(r)\right\rangle$ from the loop position to $r=b$. The interpolation requires an additional measurement, namely the azimuthal dipole field in the gap at the same radius as the radial field (i.e., on the same bore circle for the loop mounting holes, rotated by 90 degrees in theta). Integrating Eq. 3.11 from $r=b$ to the radial position of the loop, we have

$$
\begin{aligned}
r_{p}<B_{r}\left(r_{p}\right)>-b<B_{r}(b)> & =\int_{b}^{r_{p}}<B_{\theta}(r)>d r \\
& \approx\left(r_{p}-b\right)<B_{\theta}\left(r_{p}\right)>
\end{aligned}
$$

Define the (negative) ratio of the measured azimuthal field to the radial field as

$$
s=-<B_{\theta}\left(r_{p}\right)>/<B_{r}\left(r_{p}\right)>
$$

Let $\eta_{p}$ be the value measured by the loop at $r_{p}$. Then the "true" $\eta$ can be obtained from Eq. 3.12 and Eq. 3.13 as 


$$
\eta=\eta_{p}\left[1+\frac{\Delta r}{b}(1+s)\right]
$$

An additional (smaller) correction arises from the finite loop dimensions in the measurement of $\mathrm{B}_{\theta \mathrm{w}}$. The radial dependence of $B_{\theta}$ well removed from the gap region is a uniform field plus a dipole field,

$$
B_{\theta}(r)=\frac{1}{2}\left(1+\frac{b^{2}}{r^{2}}\right) B_{\theta v}
$$

The inside opening of the square RF loops used in our measurements was $\delta=3 / 4 "$, which was also the distance the loop protruded inward from the beam tube wall. Integrating the radial flux through the loop with the radial dependence given in Eq. 3.15 gives the correction factor on $\mathrm{B}_{\theta \mathrm{w}}$. This correction factor results in multiplying the RHS of Eq. 3.14 by an additional factor

$$
{ }^{c}{ }_{\theta}=(1-\delta / 2 b) /(1-\delta / b)
$$

In the standard cell ( $b=5 ")$ this factor is 1.088 ; in the injector cell $(b=7 ")$ it is 1.06 .

\subsection{Twin Lead Perturbations}

Exciting the dipole cell modes with a pair of conducting rods provides a simulation of electron beam excitation, but the EM boundary conditions are not identical. This is very similar to the excitation of a cavity mode with an RF loop. The loaded $Q$ determines the width of the resonance curve with the loop inserted in the cavity, where

$$
1 / Q_{L}=1 / Q_{0}+1 / Q_{e x t}
$$

Here, $Q_{0}$ is the "natural" $\mathrm{Q}$ of the cavity without any loop inserted, while the external $Q$ $\left(Q_{e x t}\right)$ is a measure of the power radiated away into the loop excitation circuit. The external $Q$ can be varied by changing the loop area, orientation, and output coupling impedance. To observe the "natural" mode of the cavity, the loop should be undercoupled; i.e., we should have

$$
Q_{\text {ext }} \gg Q_{0}
$$

To estimate the external $Q$ resulting from power radiated away by the twin lead, we note that the dipole magnetic field of the cell cavity will induce a net flux

$$
\Phi=\Delta \int_{-\infty}^{+\infty} B_{\perp} d z
$$


through the twin lead, where $\Delta$ is the wire spacing. The magnitude of the induced voltage is

$$
V=\omega \Phi
$$

The net power radiated away by the twin lead (in both directions) is therefore

$$
P_{\text {rad }}=\frac{V^{2}}{2 R_{L}}
$$

where the total load resistance is the series combination of the twin lead impedances looking to the right and left, twice the characteristic impedance of the twin lead $(\sim 200$ ohms) if it is matched. The external $Q$ is then

$$
\frac{1}{Q_{e x t}}=\frac{P_{r a d}}{\omega W}
$$

with $W$ being the stored energy of the mode.

We can relate this directly to the cavity mode impedance parameters since

$$
\frac{Z}{Q_{0}}=\frac{\left(\int_{-\infty}^{+\infty} B_{\perp} d z\right)^{2} c^{2}}{2 \omega W}
$$

and

$$
Z_{\perp}=\frac{\omega}{c} Q_{0}\left(\frac{Z}{Q_{0}}\right)
$$

is the peak of the resistive interaction impedance at the mode resonant frequency $\omega$.

Combining all these relations, we find the following expression for the ratio of natural $Q$ to external $Q$ :

$$
\frac{Q_{0}}{Q_{\text {ext }}}=\frac{\omega \Delta^{2} Z_{\perp}}{c R_{L}}
$$

For the twin lead parameters in our experiment, with a wire spacing of $1.5 "=3.8 \mathrm{~cm}$, the top end of the frequencies of interest $(500 \mathrm{MHz})$, and a matched $200 \mathrm{ohm}$ twin lead impedance (corresponding to $R_{L}=400 \mathrm{ohms}$ ), the external $Q$ is much larger than the natural $Q$ so long as

$$
\left|Z_{\perp}\right|<<2 \times 10^{4} \text { ohms/m }
$$




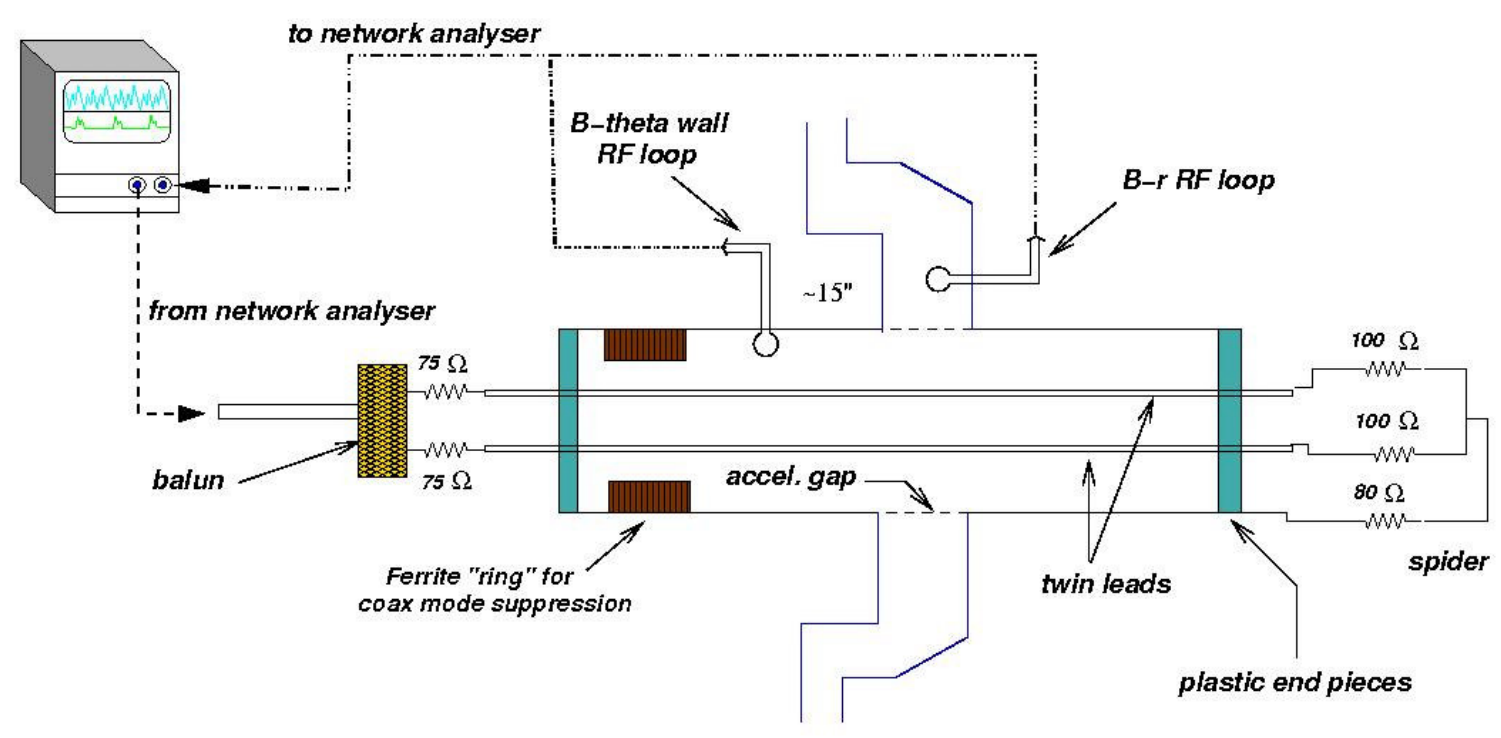

Figure 25: Schematic of the experimental configuration used in the final measurements.

The twin lead perturbation should therefore be only a few percent with our experimental parameters (when the modes are well damped).

In Ref. [5] the same upper bound on the interaction impedance for minimal twin lead perturbations was derived from a different point of view. There, the reflections from the accelerator cavity loading on the twin lead were estimated, and found to involve the same parameter as the right hand side of Eq. 3.25.

We should also point out that these considerations also apply to TSD measurements. Limitations for that technique are often more serious because the rod spacing needs to be larger to ensure adequate signal. For example, in Ref. [9] the rod spacing was $12 \mathrm{~cm}$, which makes the right hand side of Eq. 3.25 an order of magnitude larger. This can lead to significant errors in measurements of the impedance and mode $Q$ for DARHT-2 cell parameters.

\subsection{Experimental Configuration}

In this section we present more details on the experimental configuration used in the twin-lead-loop impedance measurements of the final design of the standard cell. A very similar set-up was used in the measurements of the injector cell; minor differences in the dimensions, etc., will be covered in the discussion of these measurements in Section 4.2. A schematic illustration of the set-up is presented in Fig. 25. The twin lead was constructed using two 1/2-inch diameter copper rods 61 " long, with a center-to-center spacing of 1.5". Plastic end flanges on either end of the beam tube supported the rods.

The interpretation of the experimental results assumes a pure dipole-mode traveling wave on the two-wire line, so a reasonable match to the twin-lead dipole mode is required. The practical difficulties we encountered were mainly connected in one way or the other with "RF ground loops" and the coupling of RF drive signals into the "monopole", or,++ coax mode, on the twin-lead. (Note that the induction cell itself basically "isolates" the internal 


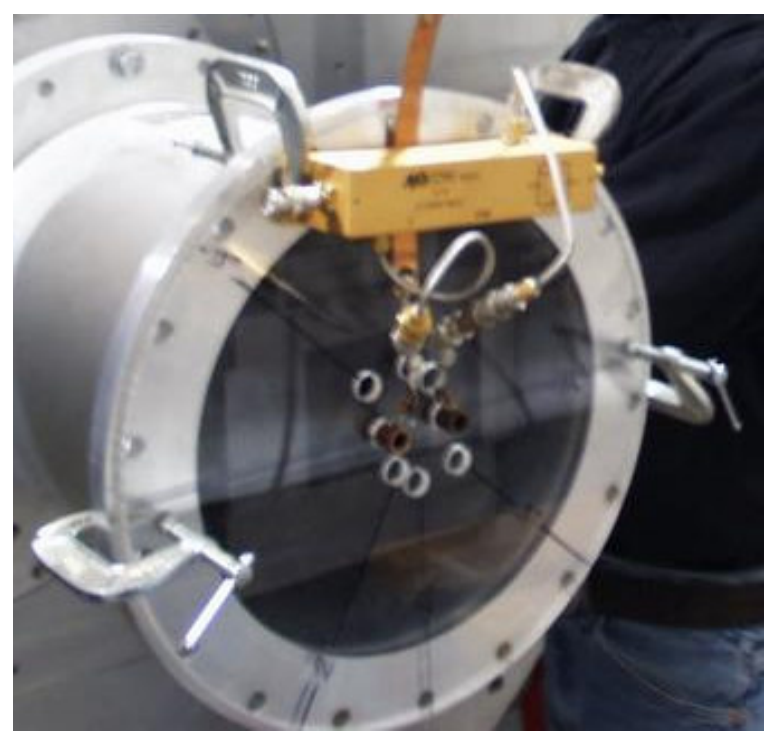

Figure 26: Photo of drive end showing the balun connections to each side of the twin leads and grounding to beam tube.

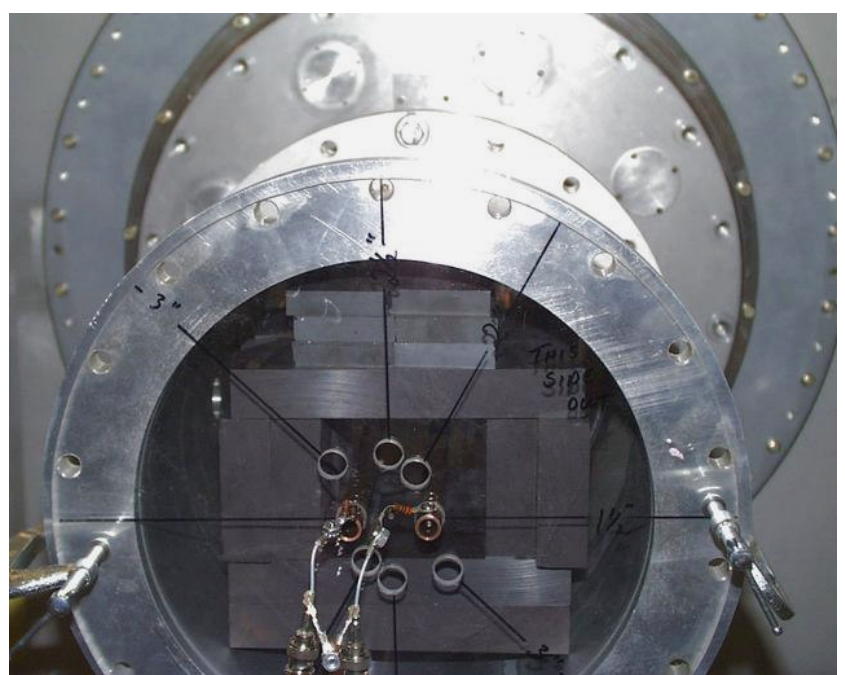

Figure 27: Photo showing ferrite blocks around twin leads used for coax mode suppression.

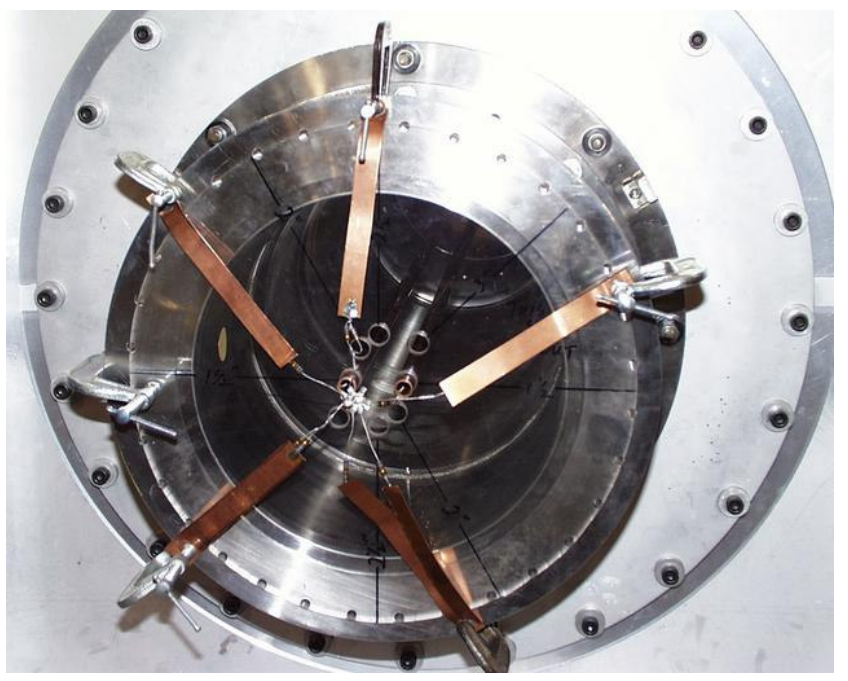

Figure 28: Photo of resistive "spider" extending from midpoint of the matched twin lead termination resistors to the beam tube wall. the twin-lead to help suppress the coax mode on the twin-lead (see the photo in Fig. 27). At the output end of the twin-lead, the best configuration we found involved connecting a resistive "spider" from the beam tube wall to the midpoint of two resistors in series that formed a matched termination ( $210 \mathrm{ohms}$ ) to the twin-lead mode (see Fig. 28). Selecting a net resistance of $\sim 80 \mathrm{ohms}$ for the resistors in parallel around this spider resulted in a termination resistance of about 140 ohms for the $(+,+)$ coax mode, close enough to its calculated characteristic impedance of 125 ohms.

A pair of RF loops on opposite sides of the beam tube (or gap), with the proper orientation to measure radial or azimuthal field components, was used to discriminate against monopole (or even $m$ ) modes. The loops were summed at a "T" junction, with 

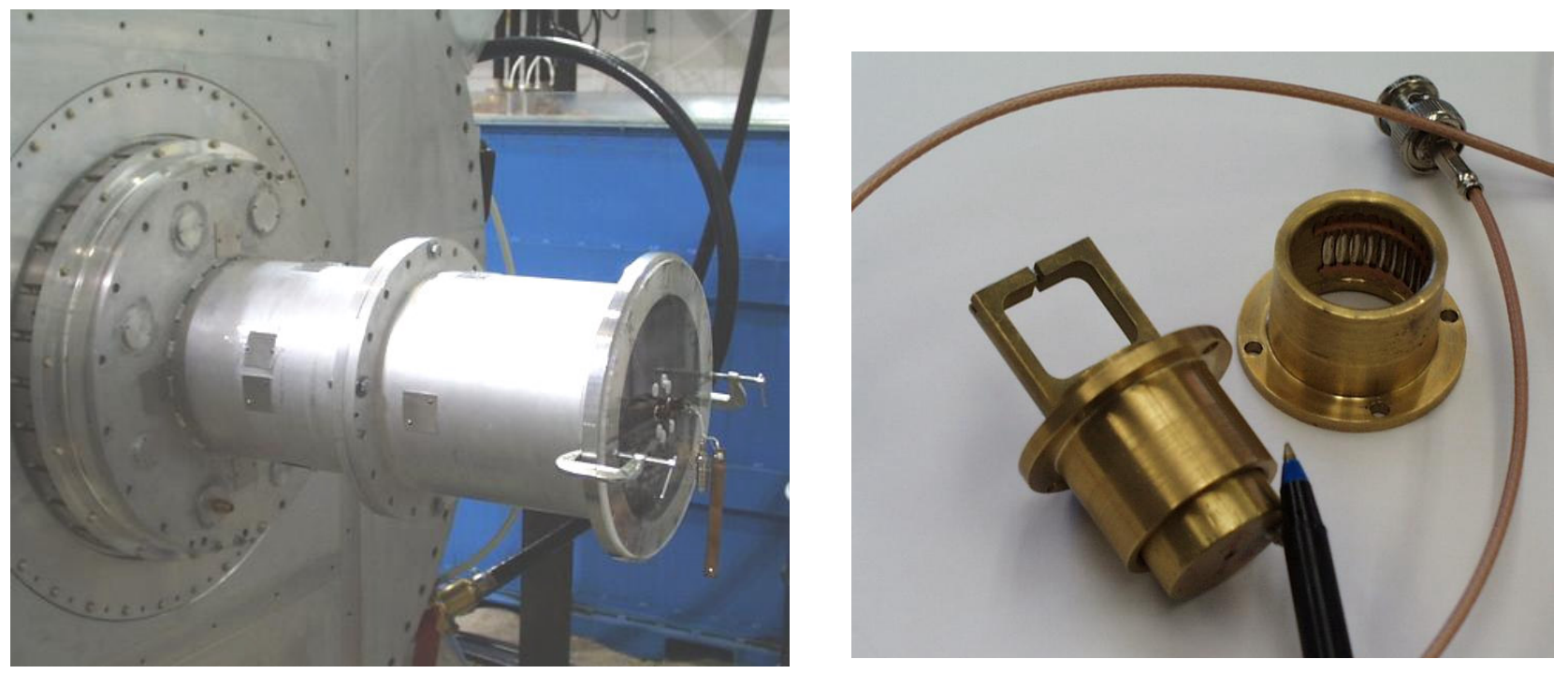

Figure 29: Drive end of beam tube showing the circular array of ports in the gap region where $B_{r}$ and $B_{\theta}$ loops were mounted, and the two openings on the beam tube (5" and 15" from the gap) where $\mathbf{B}_{\theta}$-wall loops were mounted.

$6-\mathrm{db}$ attenuators on all three sides of the junction. The locations where the loops were inserted are indicated in Fig. 29. The loops themselves are shown in Fig. 30. The shielded loop design discriminated against electric field pickup; a unique feature was construction of the loops by using machined copper with precise mechanical dimensions, an idea due to the late Dan Birx.

In making the measurements, the S12 mode of the network analyzer was used. The frequency scan of $B_{\theta w}$ was used as the reference (unity amplitude, zero phase), making the measurement of $B_{r g}$ a direct measurement of $\eta$ (when phase corrections are done, as discussed in the following Section). 


\section{Measurements of the Real and Imaginary Parts of the Impedance of the Standard Cells and Injector Cells}

The ultimate form of the twin-lead-loop measurement technique developed in the course of our campaign was described in the previous Section. In this Section, we present the results of these measurements on the 10"-standard cell and the 14"-injector cell. In each case, we also derive parameters for a three-pole resonant mode model fit to the data that could be useful in transport code modeling.

\subsection{Standard Cell Measurements and Resonant Mode Model Fit}

The experimental configuration was previously described in Section 3.4. As we noted there, the S12 network analyzer reference was set by normalizing the $B_{\theta}$-wall signal $15 "$ from the gap to unity amplitude and zero phase.

In the initial standard cell measurements reported in Section 2.6, the $B_{\theta}$-wall monitor was mounted about one beam tube radius downstream from the gap (5"). To evaluate the "purity" of the traveling wave signal set up on the twin-lead, and also to determine the errors arising from using a dipole wall current monitor this close to the gap as the reference, we measured the amplitude and phase of $B_{\theta}$-wall 5 " from the gap (10" downstream from the reference). As shown in Fig. 31, the phase difference vs. frequency is very linear up to the beam tube cutoff at $690 \mathrm{MHz}$, where we see a little "glitch". This high degree of linearity in the phase vs. frequency is consistent with a simple traveling wave; i.e., reflections from the gap and/or the termination are minimal. Above cutoff, strong deviations from linearity in phase begin, as might be expected; data in this regime are meaningless as a measure of the cell impedance.
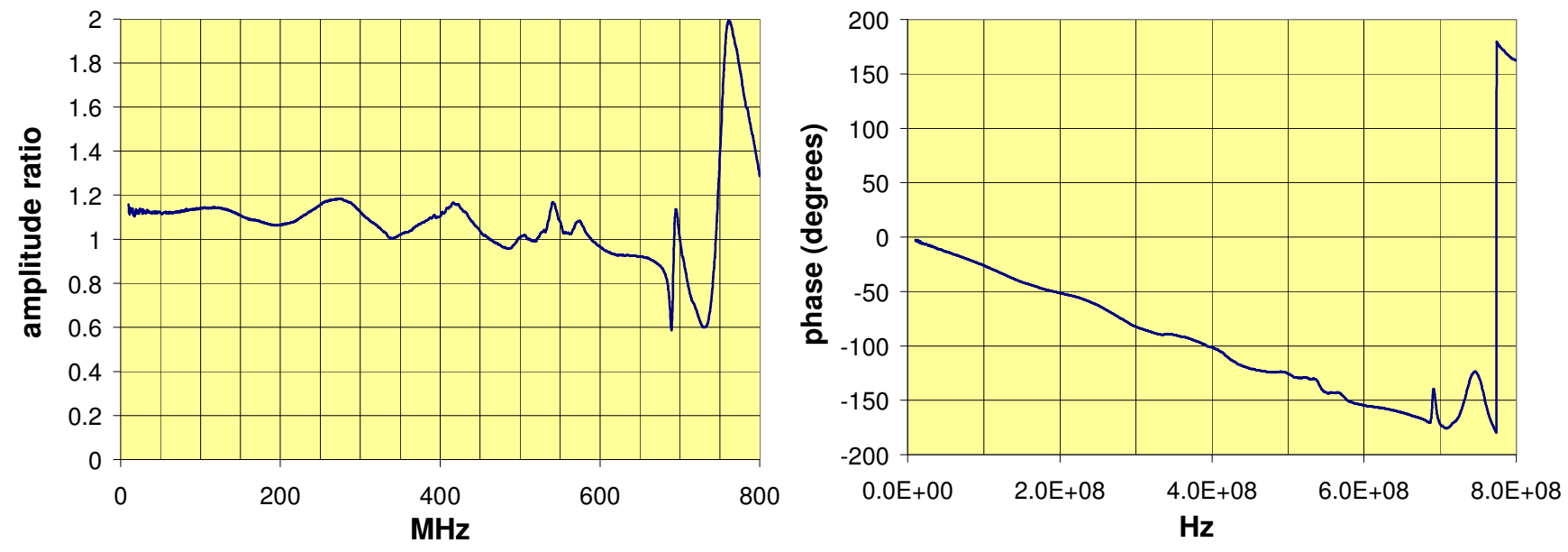

Figure 31: Linear amplitude ratio and phase difference of $B_{\theta}$-wall at 5 " to $B_{\theta}$-wall at 15 " from the acceleration gap. 
Deviations from unity in the amplitude ratio are the order of $12-15 \%$ up to the cutoff frequency. This order of magnitude is not inconsistent with the decay rate of the evanescent fields from the gap region as discussed in Section 3.1,

i.e., $\exp (-1.84 z / b)$ is about $16 \%$ at $z=b$. Above cutoff, very large deviations from unity occur, as might be expected.

In Fig. 32, the measurement of the magnitude of $B_{r}$ in the gap $\left(\eta_{p}\right)$ vs. frequency is presented (without any geometrical corrections). This result is consistent within $10 \%$ or so with the earlier measurements described in Section 2.6, within the error bars of the $B_{\theta}$-wall normalization already discussed.

Applying the geometrical corrections derived in Section 3.2, for the Standard Cell we have

$$
\eta=(1.36+0.27 s) \eta_{p}
$$

Using the measurements of $B_{\theta^{-}}$gap (s), and accounting for the phase differences in the propagation to the gap from the reference point (Eq. 3.7), we obtain the real and imaginary parts of $\eta$ as shown in Fig. 33. The resistive part of the impedance (see Eq. 3.5) is $189 \operatorname{Im}(\eta$ ) ohms/meter for the standard cell dimensions; this is shown in Fig. 34 on an expanded vertical scale.

In the calculation of BBU growth through the accelerator with computer models, the transverse interaction impedance is usually modeled by a set of discrete resonant modes, in the form

$$
\eta=\sum_{n} \frac{j \eta_{M n}}{1+j 2 Q_{n}\left(\omega-\omega_{0 n}\right) / \omega_{0 n}}+\eta_{0}
$$

For each resonant frequency, there are two free parameters: the peak value of $\operatorname{Im}(\eta)$ at resonance (related to the usual $Z_{\perp} / Q$ parameter), and the width of the resonance $(Q)$. The form in Eq. 4.2 is an approximation covering the positive frequency part of the spectrum; we consider later a more accurate representation for the full spectrum that is valid even with very low $Q$ 's.

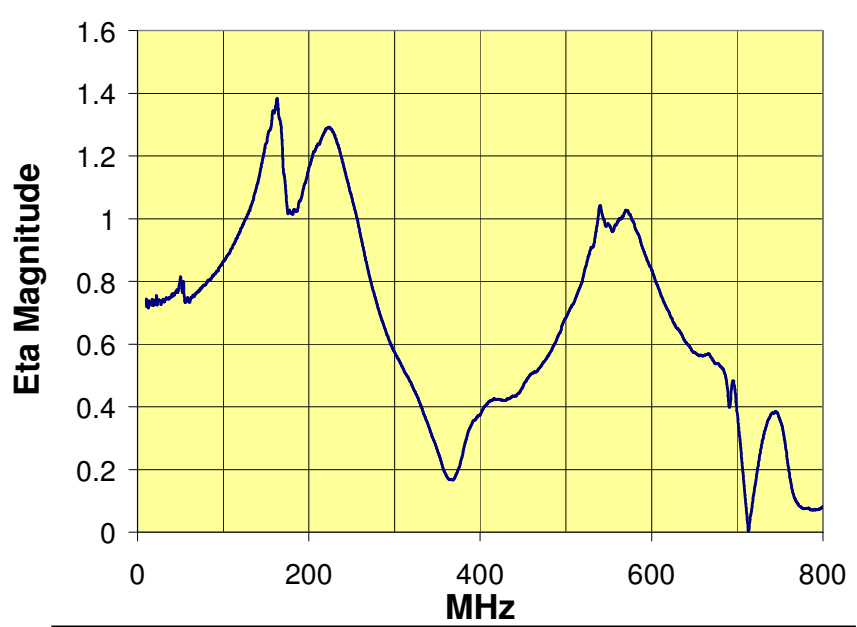

Figure 32: Uncorrected "probe " $\eta$ vs. frequency.

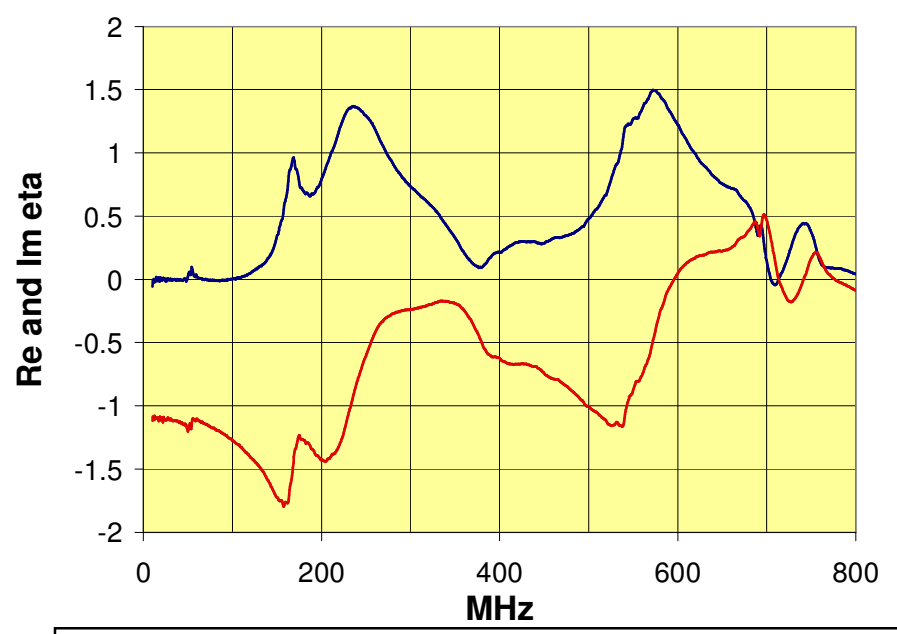

Figure 33: Real (red line, lower curve) and imaginary part of $\eta$ (blue line, upper curve) vs. frequency.

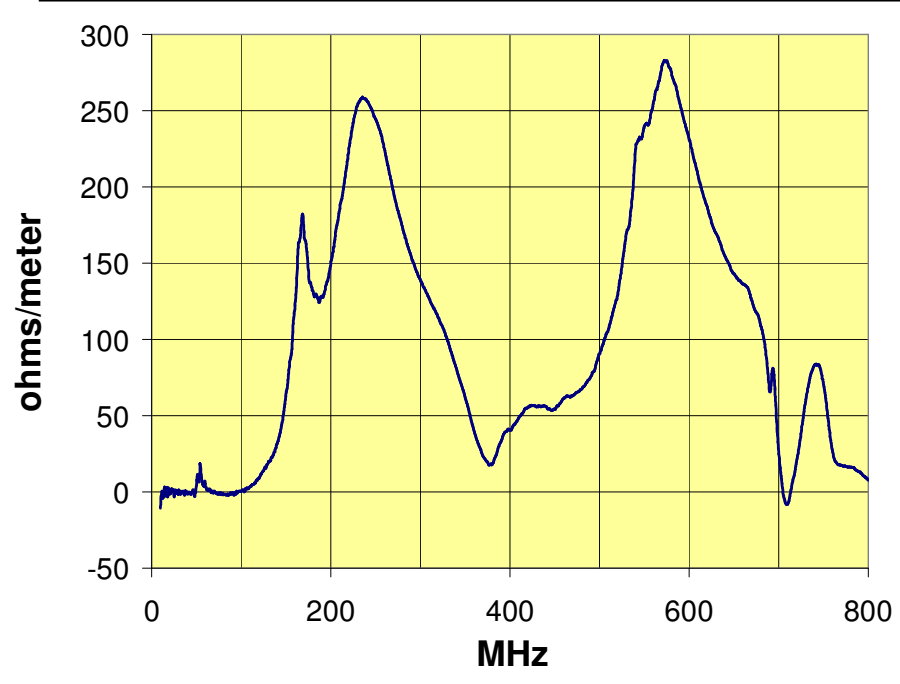

Figure 34: $Z_{\perp r}$ (ohms/meter) vs. frequency (MHz). 
To aid in the transport modeling of DARHT-2 with codes that use discrete mode representations, we did a detailed curve fitting exercise using the three most prominent resonant frequencies represented in the data in Fig. 31. The curve fitting exercise involved varying the $Q$ and $\eta_{M n}$ $(Z / Q)$ of each mode until the resistive component of the "discrete mode model" impedance was a reasonable match to the measured resistive impedance. The reactive impedance of the model is then determined, with no additional free parameters outside of a constant. We determine this constant by matching the measured low frequency limit of the reactive impedance. This

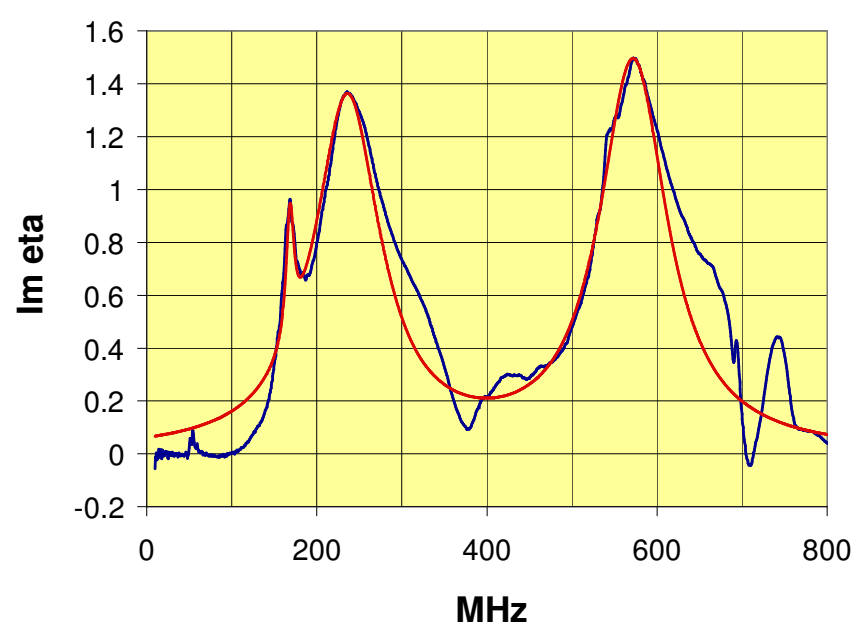

Figure 35: $\operatorname{Im}(\eta)$ - data (blue line) and fit (red line) to 3-pole resonant frequency model. interrelationship between the real and imaginary parts of impedance is well known, of course. It is also a direct analog of the Kronig-Kramers relations between real and imaginary parts of complex permittivity.

After a few iterations, necessary because of the overlap of these low $Q$ resonances, the values were chosen (see Table 3 below) to obtain a reasonable match between the model and the $\operatorname{Im}(\eta)$ vs. frequency data. (Note: we found the slope of $\operatorname{Re}(\eta)$ versus frequency at resonance to also be useful for making initial estimates of $Q$ in these iterations). The fit to the measured $\operatorname{Im}(\eta)$ obtained with these parameters is shown in Fig. 35 (red line).

As we already noted, once these parameters are chosen to fit the resistive part of the impedance, the reactive component of the impedance is then completely determined (except for a real constant). A value of $\eta_{0}=-0.7$ was chosen in the equation above to make the low frequency value of $\operatorname{Re}(\eta)$ match the data; the fit is shown in Fig. 36. The agreement is rather remarkable (up to the cutoff frequency at $690 \mathrm{MHz}$ ), especially after considering there were no free parameters left (outside of a real constant) after fitting $\operatorname{Im}(\eta)$.

The values of $Z / Q$ for each resonance in the last column are derived quantities, of course, using Eq. (2.3). These values bear little resemblance to the $Z / Q$ 's of modes in high $Q$ radial line geometries (see Section 2.2). This emphasizes again that heavily damped cells with overlapping modes are

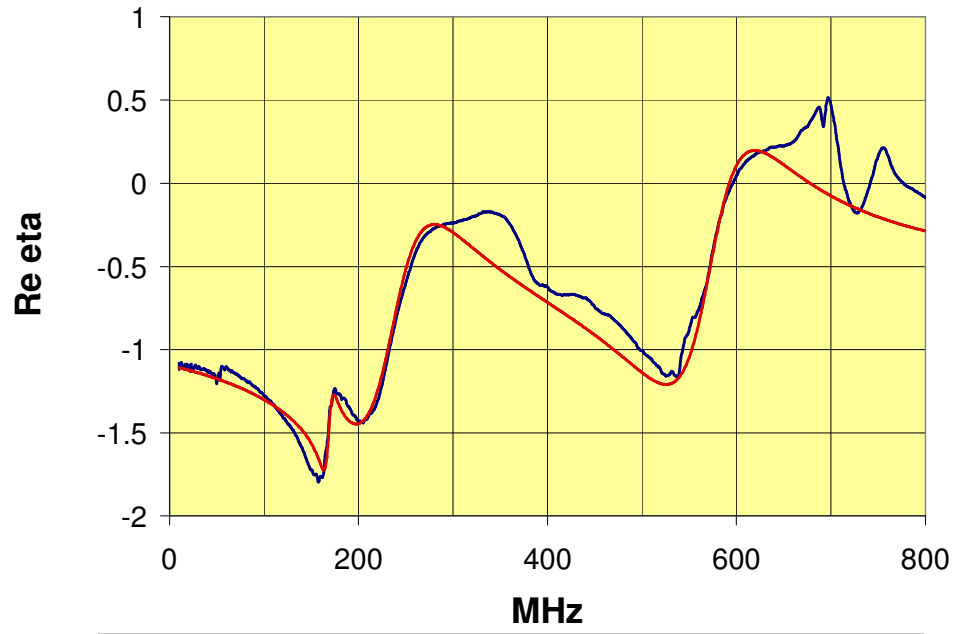

Figure 36: $\operatorname{Re}(\eta)$ - data (blue line) and fit (red line) to 3-pole resonant frequency model. poorly modeled by such high- $Q$ approximations. 


\begin{tabular}{|c|c|c|c|}
\hline $\begin{array}{c}f_{n} \text { (observed peak) } \\
(\mathrm{MHz})\end{array}$ & $\eta_{M n}$ & $Q_{n}$ & $\begin{array}{c}(Z / Q)_{n} \\
\text { (ohms) }\end{array}$ \\
\hline 168.5 & 0.49 & 15 & 1.76 \\
\hline 236 & 1.33 & 2.5 & 20 \\
\hline 572 & 1.47 & 5.88 & 4 \\
\hline
\end{tabular}

Table 3: Best-fit parameters for the 3-pole resonant frequency model

The very strong "depression" of the resistive impedance below $100 \mathrm{MHz}$ in the data is probably related to the fact that the thin ferrite slab losses drop very rapidly in this frequency regime. We should also comment that we ignored the "little biddy resonance" around $50 \mathrm{MHz}$ that appears in the data (e.g., Fig. 33). This does appear to be a "real" resonance (e.g., observe the "kink" in the phase at this frequency in the plot of $\operatorname{Re}(\eta)$ vs. $f$ in Fig. 36).

The resistive impedance of the3-pole model does not equal zero at zero frequency as it should because we left out the "negative frequency portion" in Eq. 4.2. The proper expression valid over the full spectrum is

$$
\eta=\sum_{n}\left\lfloor\frac{j \eta_{M n}}{1+j 2 Q_{n}\left(\omega-\omega_{0 n}\right) / \omega_{0 n}}-\frac{j \eta_{M n}}{1+j 2 Q_{n}\left(\omega+\omega_{0 n}\right) / \omega_{0 n}}\right\rfloor+\eta_{C}
$$

This explicitly satisfies the requirement

$$
\eta(-\omega)=\eta^{*}(+\omega)
$$

for real $\omega$, as a proper Fourier transform of a real function should.

The result of fitting this more elaborate functional form to the experimental $\operatorname{Im} \eta$ required only minor "tweeking" in the residues at the poles (given in the Table 4 below), and no changes in the $Q$ 's, for an optimized fit.

\begin{tabular}{|l|l|}
\hline$f_{n}$ (observed peak) & \multicolumn{1}{c|}{$\eta_{M n}$} \\
\hline $168.5 \mathrm{MHz}$ & $0.51(+4 \%)$ \\
\hline $236 \mathrm{MHz}$ & $1.36(+2 \%)$ \\
\hline $572 \mathrm{MHz}$ & 1.47 (no change) \\
\hline
\end{tabular}

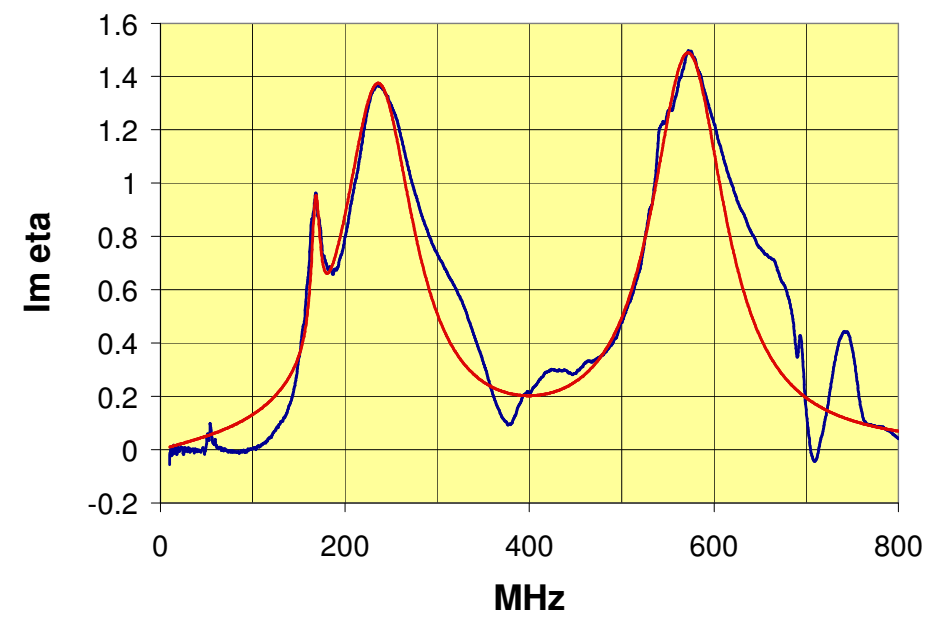

Table 4: Derived $\eta$ 's including negative freq. term

The comparison of the 3-pole "full spectrum" model with the measured $\operatorname{Im}(\eta)$ is shown in Fig. 37. The only discernable difference with the simpler model in Fig. 35 is that $\operatorname{Im}(\eta)$ now goes to zero at zero frequency (as it should). Having added the negative frequency poles, the fit of the $\operatorname{Re}(\eta)$ does require a different real constant, namely 


$$
\eta_{C}=-0.4
$$

A final question that seems appropriate to address is how well does the AMOS modeling discussed in Section 2.5 does in predicting the experimental data on the final standard cell design? The comparison between simulation and experiment is shown in Fig. 38. The most pronounced discrepancy is the absence of the "split mode" around $200 \mathrm{MHz}$ in the simulation predictions. We discuss this discrepancy in more detail in the following Section covering the injector cell measurements, where a similar difference arises.

\subsection{Injector Cell Measurements and Resonant Mode Model Fit}

The experimental setup used to measure the impedance on injector cell \#7 was very similar to that used in the standard cell measurements. The beam pipe extensions were now 14" in diameter of course, and the axial distance from the position of the $\mathrm{B}_{\theta}$-wall loop reference to the gap midplane was about 24.5", greater than 3 beam tube radii. Loop summers now had 10:1 attenuators in each leg and the "spider" at the termination end was found to be unnecessary.

With the larger beam tube diameter, the geometrical corrections are diminished; now

$$
\eta=(1.25+0.19 s) \eta_{p}
$$

The measured $\operatorname{Re}(\eta)$ and $\operatorname{Im}(\eta)$ are plotted in Fig. 39. The data below about $25 \mathrm{MHz}$ is excluded in this plot because the noise became excessive. This likely happened because the level settings on the network analyzer were not adjusted as carefully as before. The sharp "spike" around $500 \mathrm{MHz}$ is the signature of the cutoff frequency (calculated at $492 \mathrm{MHz}$ in a 14 " diameter tube). As predicted by the modeling, the upper frequency resonance seen in the standard cell has been "pushed" beyond cutoff in the injector cell. Note that the complex impedance in terms of $\eta$ for the 14-inch cells is now $Z=-96.4 j \eta$, a factor of $(1.4)^{2}$ smaller.

The injector cell impedance measurements were modeled by a set of discrete resonant modes, as

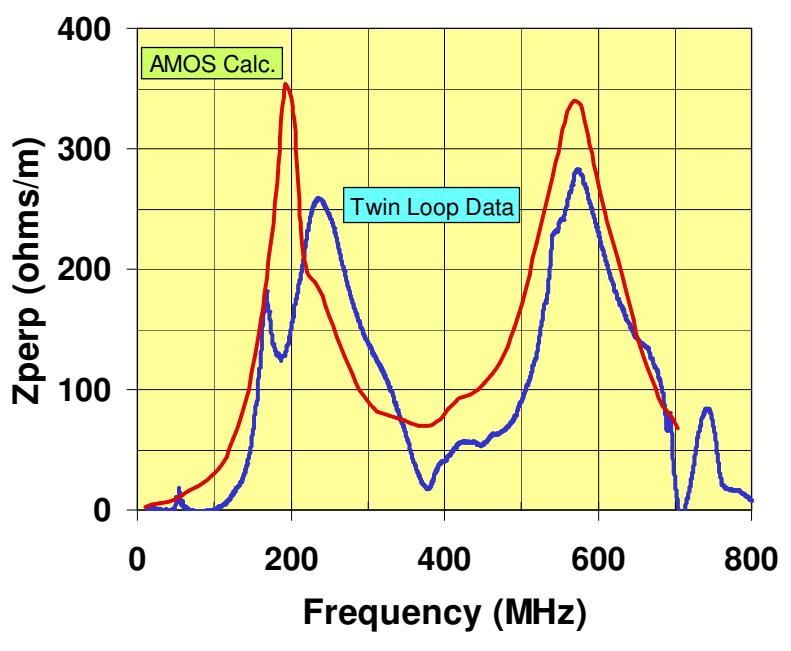

Figure 38: Comparison of AMOS calculation of the Standard Cell impedance with the Twinlead-loop measurement. AMOS does not predict the "split mode" around $200 \mathrm{MHz}$. 
before, using the representation in Eq. (4.2). Three resonant modes were used to get a reasonable fit (detailed in Table 5) to $\operatorname{Im}(\eta)$ over the frequency band up to the cutoff frequency of 492MHz:

\begin{tabular}{|c|l|l|}
\hline Frequency $f_{n}(\mathrm{MHz})$ & $\eta_{M n}$ & $Q_{n}$ \\
\hline 146 & 1.1 & 4 \\
\hline 200 & 1.25 & 2.75 \\
\hline 500 & 0.9 & 2 \\
\hline
\end{tabular}

Table 5: Injector cell fitting parameters for 3 resonant frequency model

Plots of the $\operatorname{Im}(\eta)$ data and the 3-pole model are presented in the left panel of Fig. 40. The low $Q$ mode at $500 \mathrm{MHz}$ was included to raise the resistive impedance (i.e. $\operatorname{Im}(\eta))$ values of the model in the $250-490 \mathrm{MHz}$ region to better match the data. Using the model above the cutoff frequency of $490 \mathrm{MHz}$ is an extrapolation from the measurements While this is reasonable as a broad band impedance level, it is not properly justified by the measurements which are invalid close to and above the cutoff frequency.

The parameters in Table 5 were determined from the $\operatorname{Im}(\eta)$ fit; for $\operatorname{Re}(\eta)$ we added a constant $\eta_{0}=-0.7$ to the modes to fit the zero frequency intercept (interesting that this constant is the same in the accelerator cell fitting). The data and fit for $\operatorname{Re}(\eta)$ are presented in the right panel of Fig. 40.

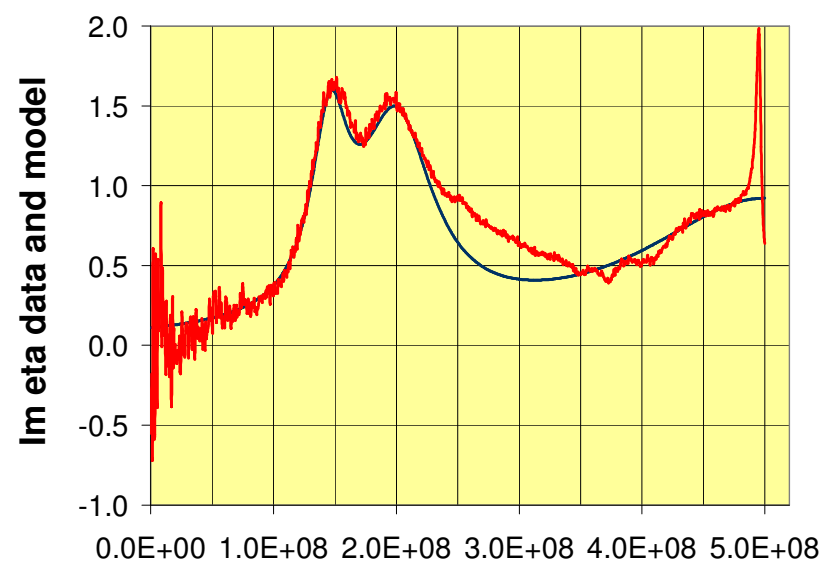

$\mathrm{MHz}$

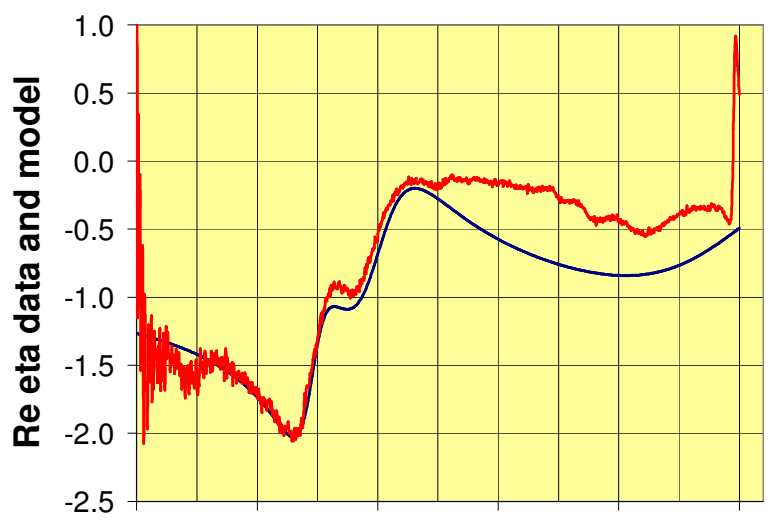

$0.0 \mathrm{E}+00 \quad 1.0 \mathrm{E}+08 \quad 2.0 \mathrm{E}+08 \quad 3.0 \mathrm{E}+08 \quad 4.0 \mathrm{E}+08 \quad 5.0 \mathrm{E}+08$

$\mathrm{MHz}$

Figure 40: $\operatorname{Im}(\eta)$ (left) and $\operatorname{Re}(\eta)$ (right) twin lead data (red line) and 3-pole resonant mode fit (blue line) for the injector cell. 
The injector cell impedance shows the same "split mode" character around $200 \mathrm{MHz}$ as did the standard cell. Since this is not predicted in either case by the AMOS code model, we searched for 3-D effects that might be responsible. The only pronounced feature along this line are the drive blades on the cells, so we removed the blades and measured the impedance. The results are shown in Fig.41. The drive blades with compensation can resistor loads do appear to kill a $50 \mathrm{MHz}$ resonance and lower the peak impedance very slightly. Nonetheless, it appears that they do not have anything to do with

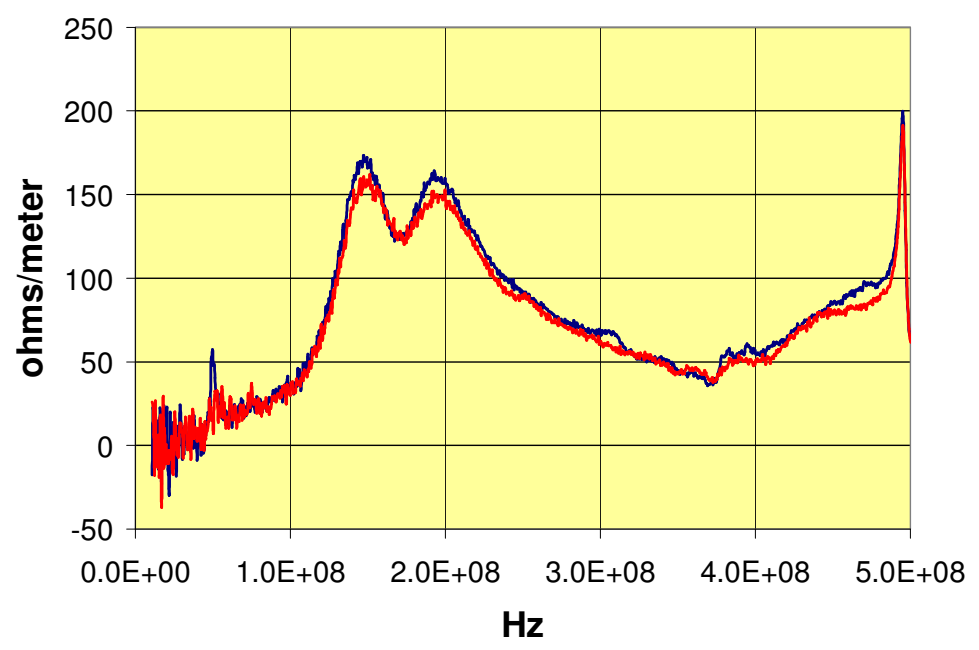

Figure 41: Measured impedance for the standard cell with drive blades (red line) and without drive blades (blue line) in the injector cells. The only significant difference is that without drive blades a stronger 50-MHz resonance appears.

the mode splitting in the $150-200 \mathrm{MHz}$ frequency.

\section{Conclusions}

By almost any measure, the campaign to minimize the DARHT-2 cell impedance was very successful. As a reference point, the measured peak impedance of the DARHT-2 standard cell $(280 \mathrm{ohms} / \mathrm{m})$ is about $1 / 3$ the measured peak impedance of the DARHT-1 cell $(880 \mathrm{ohms} / \mathrm{m}$ in the vertical direction; the average of the horizontal and vertical impedances being somewhat less). A substantial factor in this reduction comes from the larger radial bore, of course, as contained in the geometrical factor $w / b^{2}$ in the impedance (Eq. 2.2). But the "form factor" $\eta_{f}$, a useful figure of merit for how well the cell has been damped, is also less in the DARHT-2 cell (1.5 versus $\sim 2$ for DARHT-1). This is particularly noteworthy considering the fact that the massive DARHT-2 cell has a much higher dielectric constant insulator and uses Metglas rather than ferrite as the magnetic core material. In fact, the only known induction linac cell that appears to have an $\eta_{f}$ comparable to the DARHT-2 standard cell (within the accuracy of the impedance measurements at that time) is ATA $\left(\eta_{f} \sim 1.4\right)$. The optimization of the damping ferrite thickness and its long radial extent up the radial line appear to be the most important factors in the DARHT-2 cell design responsible for these results.

The elimination of high $Q$ modes is especially crucial in the long pulse regime of DARHT-2, of course, since the BBU amplification will reach the "saturated" growth limit given by Eq. (2.1) early in the pulse, even the if the BBU modes have a relatively high $Q$ (note that $Z_{\perp r}$ is proportional to $Q$ per Eq. 2.3 for "isolated" high $Q$ modes). This behavior is in contrast to short pulse machines like FXR at LLNL where the main BBU 
mode is poorly damped $(Q \sim 40)$ but the amplification does not approach the saturated growth limit until the end of the $\sim 70$-ns pulse, so consequently the damage to the radiographic spot size is less pronounced.

The development of the new "twin-lead" transverse impedance measurement technique, including a physical interpretation of the form factor and how to measure it directly, is another noteworthy outcome of the DARHT-2 cell impedance campaign. This should greatly expedite and improve the quality of the data in future impedance measurements on similar induction cells.

The AMOS computer simulations were a useful tool in assessing the sensitivity of the impedance to various geometrical changes and ferrite parameters. Unfortunately, the present code models do not have a sufficiently accurate predictive capability at this point for cells as complicated as DARHT-2. For example, the "split mode" feature so clearly displayed in the data has not been replicated. We do not have an explanation of what "physics" features might be lacking in the codes at this time. We do note that the codes do not contain any serious modeling of the RF properties of the Metglas core region.

Regarding the present (2004) effort on cell redesign for more robust voltage holding, we would not expect that the relatively "minor" changes currently planned (cathode cap, insulator triple point, hockey pucks, 1-inch longitudinal extension, etc.) will have a significant effect on the impedance. However, it is useful to recall the "surprises" we encountered in the campaign when what were perceived originally as "small changes" in the cell design led to significant quantitative changes in the impedance (as described in Section 2). Hence, we caution that an actual measurement of the impedance of the final cell configuration would seem to be prudent in any case before a final design freeze. 


\section{References}

1. R. Burgess et al. , "A Long Pulse Linac for the Second Phase of DARHT", Proc. 1999 Particle Accelerator Conference (New York, NY), 3257 (1999).

2. D. Birx and R. Briggs, " 'Corner Reflector' for RF Mode Suppression in the ATA/ETA Cells", LLNL ATA Note \#142, February 1981.

3. D.L. Birx, "Microwave Measurements of the ETA Accelerating Cavity", LLNL UCID-18582, March 1980.

4. D. Birx, R. Briggs, T. Houck, and L. Reginato, "BBU Mode Studies in a DARHT-2 Test Cell with a High Dielectric Constant Insulator", LBNL Eng. Note M7700, June 1998.

5. D. Birx, R. Briggs, and L. Reginato, "BBU Impedance Measurements on the DARHT-2 Cell", LBNL Eng Note No. 42876, February 1999.

6. J. DeFord and G. Kamin, "Application of Linear Magnetic Loss Model of Ferrite to Induction Cavity Simulation", Proc. 1990 Linear Accelerator Conference (Albuquerque, NM), 384 (1990).

7. D.J. Mayhall and S.D. Nelson, "A Comparison of AMOS Computer Code Wakefield Real Part Impedances with Analytic Results", DARHT Tech. Note No. 304, February 2003.

8. S.D. Nelson and M. Vella, "RF Cell Modeling and Experiments of Wakefield Minimization in DARHT-II", Proc. 20 ${ }^{\text {th }}$ Int. Linac Conference (Monterey, CA), 452 (2000).

9. S. D. Nelson and M. Vella, "Two Wire Wakefield Measurements of the DARHT Accelerator Cell", DARHT Technical Note No. 114, August 1999.

10. R. Briggs, D. Birx., G. Caporaso, K. Neil,and T. Genoni, "Theoretical and Experimental Investigation of the Interaction Impedances and Q Values of the Accelerating Cells in the Advanced Test Accelerator", Particle Accelerators, 18, 41 (1985).

11. B. Godfrey, "Transverse Wake Potentials for Wide Radial Lines", Mission Research Corporation MRC/ABQ-R-1046, March 1988.

12. R. Briggs, private communication (2001). 